\title{
Group Sparsity Residual Constraint for Image Denoising with External Nonlocal Self-Similarity Prior
}

\author{
Zhiyuan Zha ${ }^{a}$, Xinggan Zhang ${ }^{a}$, Qiong Wang ${ }^{a, *}$, Yechao Bai $^{a}$, Yang Chen $^{a}$, \\ Lan Tang ${ }^{a, b}$, Xin $\mathrm{Liu}^{c}$ \\ a School of Electronic Science and Engineering, Nanjing University, Nanjing 210023, \\ China. \\ ${ }^{b}$ National Mobile Commun. Research Lab., Southeast University, Nanjing 210023, China. \\ ${ }^{c}$ The Center for Machine Vision and Signal Analysis, University of Oulu, 90014, Finland.
}

\begin{abstract}
Nonlocal image representation has been successfully used in many image-related inverse problems including denoising, deblurring and deblocking. However, most existing methods only consider the nonlocal self-similarity (NSS) prior of degraded observation image, and few methods use the NSS prior from natural images. In this paper we propose a novel method for image denoising via group sparsity residual constraint with external NSS prior (GSRC-ENSS). Different from the previous NSS prior-based denoising methods, two kinds of NSS prior (e.g., NSS priors of noisy image and natural images) are used for image denoising. In particular, to enhance the performance of image denoising, the group sparsity residual is proposed, and thus the problem of image denoising is translated into reducing the group sparsity residual. Because the groups contain a large amount of NSS information of natural images, to reduce the group sparsity residual, we obtain a good estimation of the group sparse coefficients of the original image by the external NSS prior based on Gaussian Mixture Model (GMM) learning, and the group sparse coefficients of noisy image are used to approximate the estimation. To combine these two NSS priors better, an effective iterative shrinkage algorithm is developed to solve the proposed GSRC-ENSS model. Experimental results demonstrate that the proposed GSRC-ENSS not only outperforms
\end{abstract}

«* Corresponding Author. E-mail: wajj131420@hotmail.com.

Preprint submitted to Neurocomputing

October 23, 2018 
several state-of-the-art methods, but also delivers the best qualitative denoising results with finer details and less ringing artifacts.

Keywords: Image denoising, group sparsity residual constraint, nonlocal self-similarity, Gaussian Mixture Model, iterative shrinkage algorithm.

\section{Introduction}

Image denoising is not only an important problem in various image processing studies, but also an idea test bed for measuring the statistical image modeling techniques. It has attracted a lot of research interest in the past few decades [1, 2, 13, 4, 15, 6, 17, 8, 9, 10, 11, 12, 13, 14, 15, 16, 17, 18. Image denoising aims to estimate the latent clean image $\boldsymbol{X}$ from its noisy observation $\boldsymbol{Y}=\boldsymbol{X}+\boldsymbol{V}$, where $\boldsymbol{V}$ is usually assumed to be additive white Gaussian noise. Due to the ill-posed nature of image denoising, it is critical to exploit the prior knowledge that characterizes the statistical features of the images.

Previous models mainly employed the priors on level of pixel, such as Tikhonov regularization [19, total variation (TV) regularization [2]. These methods are effective in removing the noise artifacts but smear out details and tend to oversmooth the images.

Recently, patch-based prior has shown promising performance in image denoising. One representative example is sparse coding based scheme, which assumes that each patch of an image can be precisely modeled as a sparse linear combination of basic elements. These elements are called atoms and they compose a dictionary. As such, one key issue of sparse coding based scheme is to train a dictionary from natural images. Popular techniques for dictionary learning are KSVD [20], ODL [21] and SDL [22]. Gaussian mixture models (GMMs) have been successfully employed as prior for natural image patches [7, 8] and its connections with block sparse representation have been explored [8]. In [8], it is observed that the estimation of an image patch from its corresponding noisy observation under GMM model with $K$ Gaussian components. However, patch-based model usually suffers from some limits, such as great computational 
complexity, neglecting the relationship among similar patches [23, 24].

Inspired by the fact that natural images contain a large number of mutually similar patches at different locations, this so-called nonlocal self-similarity (NSS) prior was initially utilized in the work of nonlocal means denoising [1]. Due to its effectiveness, a large amount of further developments [11, 12, 13, 14, 23, 24] have been proposed. For instance, a very popular method is BM3D [12], which exploited nonlocal similar 2D image patches and 3D transform domain collaborative filtering. In [13, Marial et al. advanced the idea of NSS by group sparse coding. LPG-PCA 14 utilized nonlocal similar patches as data samples to estimate statistical parameters for PCA training. However, all the methods above only exploited the NSS prior of noisy image. In other words, for a given patch, its similar patches are collected only from degraded input, which makes the quality of image denoising largely depend on the input itself.

In fact, most existing methods only focused on the input but ignored the NSS prior of clean natural images. As we know, the information from image dataset with a large number of clean images is far richer than the noisy input image. Mention that the NSS prior from natural images was firstly developed in [11, and achieved state-of-the-art denoising performance, but how to explicitly learn the priors from input and natural images is still an open issue. To the best of our knowledge, few works have exploited the NSS priors from both input and natural images.

With the above considerations, in this work we propose a novel method for image denoising via group sparsity residual constraint with external nonlocal NSS prior (GSRC-ENSS). The major contributions of this paper are summarized as follows. First, to improve the performance of image denoising, we propose the concept of group sparsity residual, and thus the problem of image denoising is turned into reducing the group sparsity residual. Second, due to the fact that the groups contain a large amount of NSS information of natural images, to reduce the group sparsity residual, we obtain a good estimation of the group sparse coefficients of the original image by the NSS prior of natural images based on Gaussian Mixture Model (GMM) learning, and the group sparse coefficients 
of noisy image are used to approximate the estimation. The flowchart of the proposed method is illustrated in Fig. 1. To fuse these two NSS priors better, we present an effective iterative shrinkage algorithm to solve the proposed GSRCENSS model. Our experimental results demonstrate that the proposed GSRCENSS model outperforms many state-of-the-art methods. What's more, the proposed GSRC-ENSS delivers the best qualitative denoising results with finer details and less ringing artifacts.

The reminder of this paper is organized as follows. Section 2 provides a brief survey of related work. Section 3 introduces the modeling of group sparsity residual constraint (GSRC), learns the NSS prior from natural image by GMM and presents an effective iterative shrinkage algorithm to solve the GSRC-ENSS model. Section 4 discusses the main difference among the GSRC-ENSS method, the NCSR method [25], most existing NSS prior-based denoising methods and the popular methods combining external and internal priors. Section 5 presents the experimental results. Finally, several concluding remarks are given in Section 6 .

\section{Related Work}

Image denoising is a typical ill-posed inverse problem. To solve such ill-posed problem, it is of great importance to exploit the prior knowledge we know about the original image so that we can regularize the solution and various image prior models have been developed [1, 2, 3, 4, 5, 6, 7, 8, 9, 10, 11, 12, 13, 14, 15, 16, 17, 18 .

Early prior models mainly consider the local correlation among image pixels. One representative work is the total variation (TV) regularization method [2], which usually uses the local structure information and high effectiveness to recover the smooth regions. The TV regularization method actually assumes that natural image gradients have a heavy-tailed distribution, which can be fitted by Laplacian or hyper-Laplacian models [26, 27]. In addition, many statistical models of wavelet coefficients have also been proposed, such as generalized 


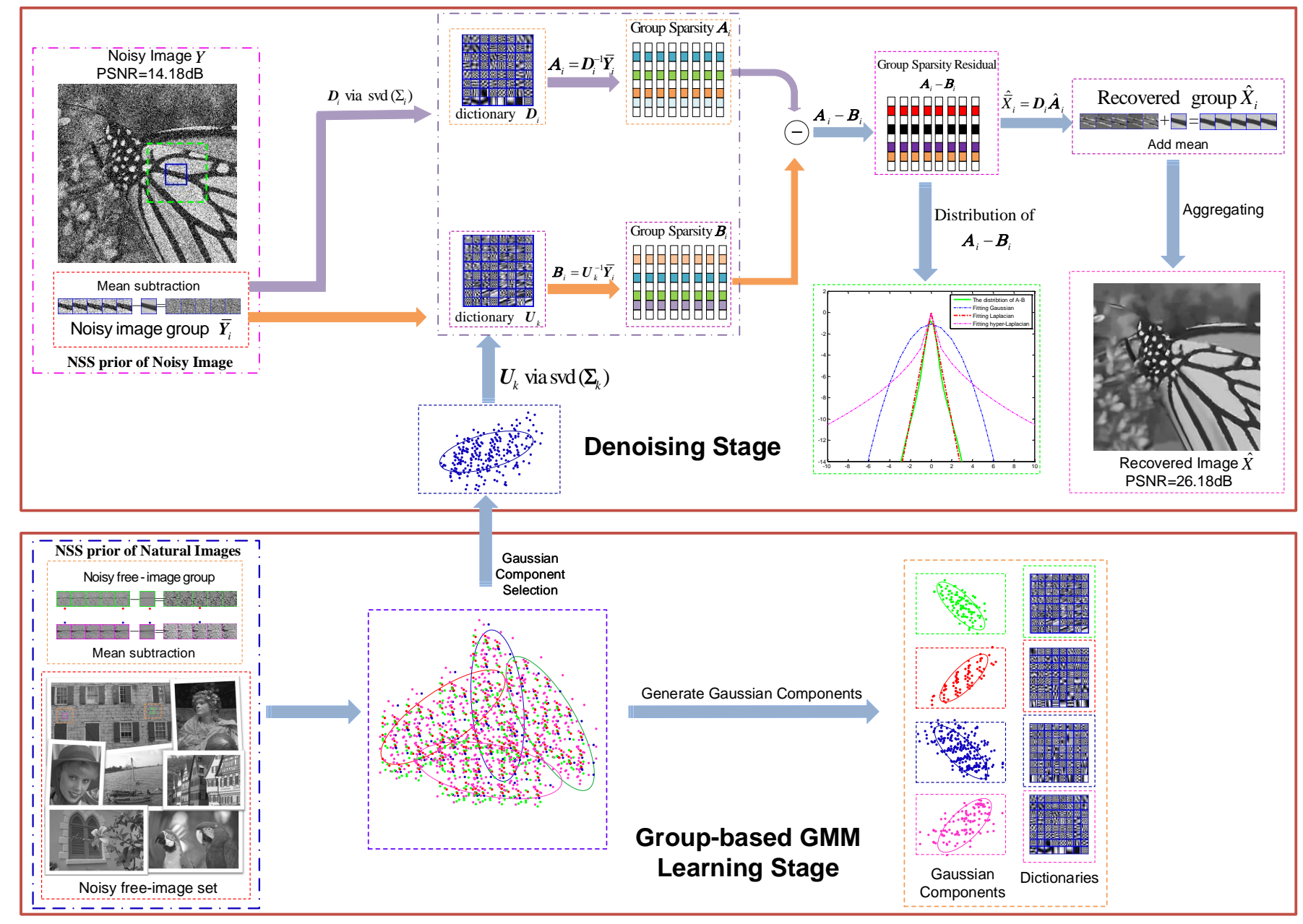

Figure 1: Flowchart of image denoising by group sparsity residual constraint with external NSS prior. 
Gaussian 28] and Gaussian scale mixture models [29, etc.

Besides the above prior models, another significant property of natural images is to model the prior on patches. One representative example is sparse coding based scheme [6, 20, which assumes that each patch of an image can be perfectly represented by a sparse coefficient vector whose entries are mostly zero or close to zero based on a basis set called as dictionary. The dictionary is usually learned from a natural image dataset 6, 20. Compared with the conventional analytically designed dictionaries, such as those based on wavelet, curvelet and DCT, dictionaries learned directly from images have an advantage of being better adapted to image local structures [30, and thus could enhance the sparsity which leads to better performance. For instance, the seminal of KSVD dictionary learning [20] has not only achieved promising denoising performance, but also extended and successfully exploited it in various image processing and computer vision tasks [31, 32, 33, 34].

Considering that natural images are non-Gaussian and image patches are regarded as samples of a multivariate vector, Gaussian mixture models (GMMs) have emerged as favored prior for natural image patches in various image restoration studies [7, 8, 9. For instance, Zoran et al. 7] exploited GMM model to learn image patches from clean natural image dataset, and proposed a method which can reconstruct the latent image by maximizing the expected patch log likelihood. Yu et al. [8] estimated the latent clean image from its noisy observation under GMM model with $K$ Gaussian components. Niknejad et al. 9] used GMM model with spatially constraint patch clustering to recover the latent image from noisy image.

Motivated by the fact that image patches that have similar structures can be spatially far from each other and thus can be collected in the whole image, this so-called nonlocal self-similarity (NSS) prior is among the most remarkable priors for image restoration. The seminal work of nonlocal means (NLM) [1] utilized the NSS prior to implement a form of the weighted filtering for image denoising. After this, inspired by the success of the NLM denoising filtering, a flurry of NSS based methods [11, 12, 13, 14, 23, 24, 25, 27, 30, 35] were 
proposed to solve various image inverse problems. Among these NSS based schemes, the famous BM3D method [12] exploited nonlocal similar patches and 3D transform domain collaborative filtering, which can achieve state-of-theart performance in image denoising. Dong et al. 25] proposed the nonlocally centralized sparse representation (NCSR) model for image restoration, which obtained sparse coding coefficients estimate of the original image by the principle of NLM [1, and then according to those estimates, NCSR, centralized the sparse coding coefficients of the observed image to improve the denoising performance. $\mathrm{Xu}$ et al. 11] proposed a patch group based on NSS scheme to learn NSS models from natural images for high performance denosing.

Motivated by the observation that the nonlocal similar patches (forming a matrix) in a natural image are linearly correlated with each other, in [24, 36, 37, the authors transformed the image denoising into the problem of low rank matrix approximation, which achieved highly competitive recovery results.

Different image priors describe different and complementary aspects of natural image statistics, and it is possible to combine multiple priors to improve the denoising performance. For instance, Zhang et al. 38] proposed joint statistical modeling for various image inverse problems. Mosseri et al. [39] exploited a patch-SNR as a metric to decide whether a noisy patch is denoised using internal priors or external priors. Yue et al. [40] used a frequency domain method to fuse the internal and external denoising results. Lu et al. [41] proposed a unified method that learns a generic prior from external images and adapts the prior to a specific images. Luo et al. [42] proposed a prior learning algorith$\mathrm{m}$ that combines the internal and external priors from a Bayesian hyper-prior perspective.

Very recently, deep learning based methods have been proposed for image denoising tasks [43, 44, 45, 46, 47, 48. For example, Jain et al. 43] proposed to use convolutional neural network (CNN) for natural image denoising and claimed that CNN has similar or even better representation power than Markov random field (MRF) model [49. Burger et al. 45] proposed a patch-based denoising algorithm that is learned on a large dataset with a plain neural network. 
Liu et al. 46] considered the denoising problem as recursive image filtering via a hybrid neural network. Mao et al. [48] proposed a very deep fully convolutional encoding-decoding framework for image denoising.

\section{Group Sparsity Residual Constraint for Image Denoising with Ex- ternal Nonlocal Self-Similarity Prior}

As we know, a majority of existing methods only consider the nonlocal selfsimilarity (NSS) prior of noisy input image, and few methods use the NSS prior from natural images. In this section, two kinds of NSS prior (i.e., NSS priors of noisy image and natural images) are exploited for image denoising. To improve the performance of image denoising, we propose the concept of group sparsity residual, and thus the problem of image denoising is turned into reducing the group sparsity residual. To this end, a good estimation of the group sparse coefficients of the original image are obtained by the external NSS prior based on GMM learning, and the group sparse coefficients of noisy image are used to approximate the estimation. To fuse these two NSS priors better, we present an iterative shrinkage algorithm to solve the proposed scheme.

\subsection{Group-based sparse coding}

Recent advances [12, 13, 23, 50, 51, 52, have suggested that structured or group sparsity can offer powerful reconstruction performance for image denoising. More specifically, given a clean image $\boldsymbol{X}$, for each image patch $\boldsymbol{x}_{i}$ of size $d \times d$ in $\boldsymbol{X}, i=1,2, \ldots, n$, its $m$ best matched patches are selected from a $W \times W$ sized search window to form a group $\boldsymbol{X}_{i}$, denoted by $\boldsymbol{X}_{i}=\left\{\boldsymbol{x}_{i, 1}, \boldsymbol{x}_{i, 2}, \ldots, \boldsymbol{x}_{i, m}\right\}$, where $\boldsymbol{x}_{i, m}$ denotes the $m$-th similar patch (column vector) of the $i$-th group. Similar to patch-based sparse coding [6, 20, given a dictionary $\boldsymbol{D}_{i}$, each group $\boldsymbol{X}_{i}$ can be sparsely represented as $\boldsymbol{B}_{i}=\boldsymbol{D}_{i}^{-1} \boldsymbol{X}_{i}$ and solved by the following $\ell_{p}$-norm minimization problem,

$$
\boldsymbol{B}_{i}=\arg \min _{\boldsymbol{B}_{i}}\left\{\left\|\boldsymbol{X}_{i}-\boldsymbol{D}_{i} \boldsymbol{B}_{i}\right\|_{F}^{2}+\lambda_{i}\left\|\boldsymbol{B}_{i}\right\|_{p}\right\}
$$


where $\|\bullet\|_{F}^{2}$ denotes the Frobenious norm, $\lambda_{i}$ is the regularization parameter, and $p$ characterizes the sparsity of $\boldsymbol{B}_{i}$. Then the whole image $\boldsymbol{X}$ can be represented by the set of group sparse codes $\left\{\boldsymbol{B}_{i}\right\}$.

In image denoising, each noise patch $\boldsymbol{y}_{i}$ is extracted from the noisy image $\boldsymbol{Y}$. Like in the above paragraph, we search for its similar patches to generate a group $\boldsymbol{Y}_{i}$, i.e., $\boldsymbol{Y}_{i}=\left\{\boldsymbol{y}_{i, 1}, \boldsymbol{y}_{i, 2}, \ldots, \boldsymbol{y}_{i, m}\right\}$. Thus, image denoising is turned into how to reconstruct $\boldsymbol{X}_{i}$ from $\boldsymbol{Y}_{i}$ by using group sparse coding,

$$
\boldsymbol{A}_{i}=\arg \min _{\boldsymbol{A}_{i}}\left\{\left\|\boldsymbol{Y}_{i}-\boldsymbol{D}_{i} \boldsymbol{A}_{i}\right\|_{F}^{2}+\lambda_{i}\left\|\boldsymbol{A}_{i}\right\|_{p}\right\}
$$

Once all group sparse codes $\left\{\boldsymbol{A}_{i}\right\}$ are obtained, the latent clean image $\boldsymbol{X}$ can be reconstructed as $\hat{\boldsymbol{X}}=\boldsymbol{D} \boldsymbol{A}$, where the group sparse code $\boldsymbol{A}$ includes the set of $\left\{\boldsymbol{A}_{i}\right\}$.

\subsection{Group Sparsity Residual Constraint}

Although group sparsity has demonstrated its effectiveness in image denoising, due to the influence of noise, it is very difficult to estimate the true group sparse code $\boldsymbol{B}$ from noisy image $\boldsymbol{Y}$. That is, the group sparse code $\boldsymbol{A}$ achieved by solving Eq. (2) is expected to be close enough to the true group sparse code $\boldsymbol{B}$ of the original image $\boldsymbol{X}$ in Eq. (1). Therefore, the quality of image denoising largely depends on the level of the group sparsity residual, which is defined as the difference between group sparse code $\boldsymbol{A}$ and true group sparse code $\boldsymbol{B}$,

$$
\boldsymbol{R}=\boldsymbol{A}-\boldsymbol{B}
$$

Therefore, to reduce the group sparsity residual $\boldsymbol{R}$ and enhance the accuracy of $\boldsymbol{A}$, we propose the group sparsity residual constraint (GSRC) [53] to image denoising, and thus Eq. 22 can be rewritten as

$$
\boldsymbol{A}_{i}=\arg \min _{\boldsymbol{A}_{i}}\left\{\left\|\boldsymbol{Y}_{i}-\boldsymbol{D}_{i} \boldsymbol{A}_{i}\right\|_{F}^{2}+\lambda_{i}\left\|\boldsymbol{A}_{i}-\boldsymbol{B}_{i}\right\|_{p}\right\}
$$

However, it can be seen that the true group sparse code $\boldsymbol{B}$ and $p$ are unknown because the original image $\boldsymbol{X}$ is not available. We now discuss how to obtain $\boldsymbol{B}$ and $p$. 


\subsection{Learning the NSS Prior from Natural Images by GMM}

Since the original image $\boldsymbol{X}$ is not available, it seems to be difficult to obtain the true group sparse code $\boldsymbol{B}$. Nonetheless, we can obtain some good estimation of $\boldsymbol{B}$. Generally speaking, there are various methods to estimate the true group sparse code $\boldsymbol{B}$, which depends on prior knowledge of the original image $\boldsymbol{X}$ we have. In recent years, patch-based or group-based priors referring to denoising operators learned from natural images achieved the state-of-the-art denoising results [6, 7, 11, 20]. For instance, in 6], a dictionary learning-based method is introduced for compact patch representation, whereas in [11, a GMM model is learned from natural image groups based on NSS scheme and used as a prior for denoising. Due to the fact that the groups contain a rich amount of NSS information of natural images, we can achieve the group sparse code $\boldsymbol{B}$ by the NSS prior of natural images based on GMM learning.

Like in subsection 3.1 , we extract $N$ groups from a given clean natural image dataset, and we denote one group as

$$
\overline{\boldsymbol{Z}}_{i}=\left\{\overline{\boldsymbol{z}}_{i, j}\right\}_{j=1}^{m}, i=1,2, \ldots, N
$$

where $\overline{\boldsymbol{Z}}_{i}{ }^{1}$ is the group mean substraction of each group $\boldsymbol{Z}_{i}$ and $\overline{\boldsymbol{z}}_{i, j}$ denotes the $j$-th similar patch (column vector) of the $i$-th group. Since GMM has been successfully used to model the image patch or group priors such as EPLL [7], PLE [8] and PGPD [11, we adopt the strategy in [11] and learn a finite GMM over natural image groups $\left\{\overline{\boldsymbol{Z}}_{i}\right\}$ as group priors. By using the GMM model, the likelihood of a given group $\left\{\overline{\boldsymbol{Z}}_{i}\right\}$ is:

$$
P\left(\overline{\boldsymbol{Z}}_{i}\right)=\sum_{k=1}^{K} \pi_{k} \prod_{j=1}^{m} \mathcal{N}\left(\overline{\boldsymbol{z}}_{i, j} \mid \boldsymbol{\mu}_{k}, \boldsymbol{\Sigma}_{k}\right)
$$

where $K$ is the total number of mixture components selected from the GMM, and the GMM model is parameterized by mean vectors $\left\{\boldsymbol{\mu}_{k}\right\}$, covariance matrices $\left\{\boldsymbol{\Sigma}_{k}\right\}$, and mixture weights of mixture components $\left\{\pi_{k}\right\}$. By assuming

\footnotetext{
${ }^{1}$ The advantage of group mean substraction is that it can further facilitate the NSS prior learning because the possible number of patterns is reduced, while the training samples of each pattern are increased.
} 
that all the groups are independent, the overall objective likelihood function is $\mathcal{L}=\prod_{i=1}^{N} P\left(\overline{\boldsymbol{Z}}_{i}\right)$. Then by applying log to it, we maximize the objective function as:

$$
\ln \mathcal{L}=\sum_{i=1}^{N} \ln \left(\sum_{k=1}^{K} \pi_{k} \prod_{j=1}^{m} \mathcal{N}\left(\overline{\boldsymbol{z}}_{i, j} \mid \boldsymbol{\mu}_{k}, \boldsymbol{\Sigma}_{k}\right)\right)
$$

We collectively represent three parameters $\boldsymbol{\mu}_{k}, \boldsymbol{\Sigma}_{k}$ and $\pi_{k}$ by $\boldsymbol{\Theta}=\left\{\boldsymbol{\mu}_{k}, \boldsymbol{\Sigma}_{k}, \pi_{k}\right\}_{k=1}^{K}$, and $\Theta$ is learned using Expectation Maximization algorithm (EM) [54].

In the E-step, we calculate the posterior probability for the component $k$ as:

$$
\begin{aligned}
P\left(k \mid \overline{\boldsymbol{z}}_{i, j}, \boldsymbol{\Theta}\right) & =\frac{\pi_{k} \prod_{j=1}^{m} \mathcal{N}\left(\overline{\boldsymbol{z}}_{i, j} \mid \boldsymbol{\mu}_{k}, \boldsymbol{\Sigma}_{k}\right)}{\sum_{l=1}^{K} \pi_{l} \prod_{j=1}^{m} \mathcal{N}\left(\overline{\boldsymbol{z}}_{i, j} \mid \boldsymbol{\mu}_{l}, \boldsymbol{\Sigma}_{l}\right)} \\
N_{k} & =\sum_{i=1}^{N} P\left(k \mid \overline{\boldsymbol{z}}_{i, j}, \boldsymbol{\Theta}\right)
\end{aligned}
$$

In the M-step, for each group $\overline{\boldsymbol{Z}}_{i}$, we update the model parameters as follows:

$$
\begin{gathered}
\pi_{k}=N_{k} / N \\
\boldsymbol{\mu}_{k}=\frac{\sum_{i=1}^{N} \pi_{k} \sum_{j=1}^{m} \overline{\boldsymbol{z}}_{i, j}}{\sum_{i=1}^{N} \pi_{k}} \\
\boldsymbol{\Sigma}_{k}=\frac{\sum_{i=1}^{N} P\left(k \mid \overline{\boldsymbol{z}}_{i, j}, \Theta\right) \overline{\boldsymbol{z}}_{i, j} \overline{\boldsymbol{z}}_{i, j}^{T}}{N_{k}}
\end{gathered}
$$

We iterate over the E-Step and M-Step until convergence. For more details about EM algorithm, please refer to [54].

Thus, for each noisy group $\boldsymbol{Y}_{i}{ }^{2}$ of noisy image $\boldsymbol{Y}$, the best suitable Gaussian component is selected from this group-based GMM learning stage. To be concrete, assume that the image is corrupted by the Gaussain white noise with variance $\sigma^{2}$, then the covariance matrix of the $k$-th Gaussian component will

\footnotetext{
${ }^{2}$ In this work, all noisy groups are preprocessed by mean substraction. The mean $\boldsymbol{\mu}_{i}$ of each noisy group $\boldsymbol{Y}_{i}$ is very close to the mean of the original group $\boldsymbol{X}_{i}$ because the mean of noise $\boldsymbol{V}$ is nearly zero. Thus, the mean $\boldsymbol{\mu}_{i}$ can be added back to the denoised group $\hat{\boldsymbol{X}}_{i}$ to achieve the latent clean image $\hat{\boldsymbol{X}}$.
} 
turn into $\boldsymbol{\Sigma}_{k}+\sigma^{2} \boldsymbol{I}$, where $\boldsymbol{I}$ represents the identity matrix. The selection that $\boldsymbol{Y}_{i}$ belongs to the $k$-th Gaussian component can be accomplished by computing the following posterior probability,

$$
P\left(k \mid \boldsymbol{Y}_{i}\right)=\frac{\prod_{j=1}^{m} \mathcal{N}\left(\boldsymbol{y}_{i, j} \mid \mathbf{0}, \boldsymbol{\Sigma}_{k}+\sigma^{2} \boldsymbol{I}\right)}{\sum_{l=1}^{K} \prod_{j=1}^{m} \mathcal{N}\left(\boldsymbol{y}_{i, j} \mid \mathbf{0}, \boldsymbol{\Sigma}_{l}+\sigma^{2} \boldsymbol{I}\right)}
$$

We maximize it, and finally, the Gaussian component with the highest probability is selected to operate each group $\boldsymbol{Y}_{i}$.

Then, we assume that the $k$-th Gaussian component is selected for the group $\boldsymbol{Y}_{i}$. Actually, here GMM model is equivalent to the block sparse estimation with a block dictionary having $K$ blocks wherein each block corresponds to the principle component analysis (PCA) [30] basis of one of the Gaussian components in the mixture [8, 55]. Thus, the covariance matrix of the $k$-th Gaussian component is denoted by $\boldsymbol{\Sigma}_{k}$. By using singular value decomposition to $\boldsymbol{\Sigma}_{k}$, we have

$$
\boldsymbol{\Sigma}_{k}=\boldsymbol{U}_{k} \boldsymbol{\Lambda}_{k} \boldsymbol{U}_{k}^{T}
$$

where $\boldsymbol{U}_{k}$ is an orthonormal matrix formed by the eigenvector of $\boldsymbol{\Sigma}_{k}$ and $\boldsymbol{\Lambda}_{k}$ is the diagonal matrix of eigenvalues. With the group-based GMM learning, as the statistical structures of NSS variations in natural image are captured by the eigenvectors in $\boldsymbol{U}_{k}$, thus $\boldsymbol{U}_{k}$ can be used to represent the structural variations of the groups in that component. Finally, for each group $\boldsymbol{Y}_{i}$, each true group sparse code $\boldsymbol{B}_{i}$ can be estimated by $\boldsymbol{B}_{i}=\boldsymbol{U}_{k}{ }^{-1} \boldsymbol{Y}_{i}$.

Similar to $\boldsymbol{B}_{i}$, the covariance matrix of each group $\boldsymbol{Y}_{i}$ is defined as $\boldsymbol{\Sigma}_{i}$ and we have

$$
\boldsymbol{\Sigma}_{i}=\boldsymbol{D}_{i} \boldsymbol{\Lambda}_{i} \boldsymbol{D}_{i}^{T}
$$

where $\boldsymbol{D}_{i}$ is an orthonormal matrix formed by the eigenvector of $\boldsymbol{\Sigma}_{i}$ and $\boldsymbol{\Lambda}_{i}$ is the diagonal matrix of eigenvalues. Thus, $\boldsymbol{A}_{i}$ can be solved by $\boldsymbol{A}_{i}=\boldsymbol{D}_{i}{ }^{-1} \boldsymbol{Y}_{i}$.

\subsection{How to determine $p$}

Besides estimating $\boldsymbol{B}$, we also need to determine the value of $p$. Here we perform some experiments to investigate the statistical property of $\boldsymbol{R}$, where $\boldsymbol{R}$ 


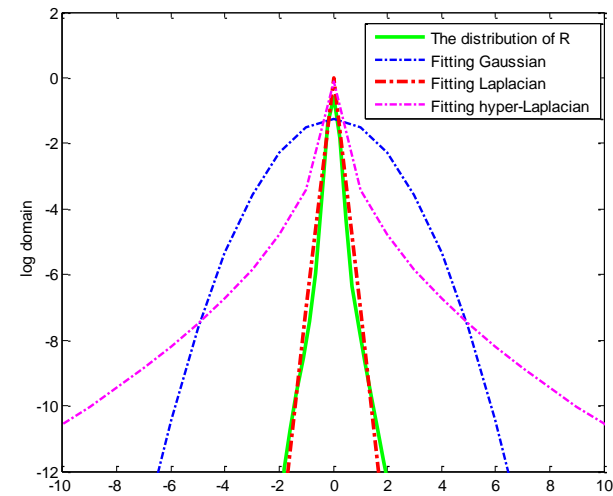

(a)

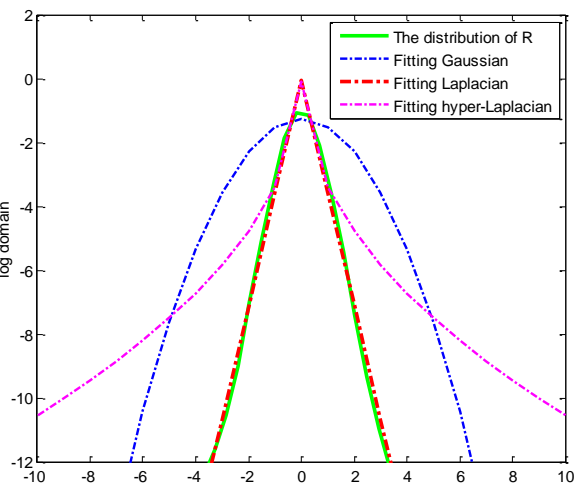

(b)

Figure 2: The distribution of $\boldsymbol{R}$, fitting Gaussian, Laplacian and hyper-Laplacian distribution for image Monarch with $\sigma=30$ in (a) and foreman with $\sigma=100$ in (b).

denotes the set of $\boldsymbol{R}_{i}=\boldsymbol{A}_{i}-\boldsymbol{B}_{i}$. In these experiments, image Monarch and foreman are used as examples, where Gaussian white noise is added to the images Monarch and foreman with standard deviation $\sigma=30$ and $\sigma=100$, respectively. We plot the histogram of $\boldsymbol{R}$ as well as the fitting Gaussian, Laplacian and hyperLaplacian distribution in the log domain in Fig. 2(a) and Fig. 2(b), respectively. It can be seen that the histogram of $\boldsymbol{R}$ can be well characterized by the Laplacian distribution. Thus, the $\ell_{1}$-norm is adopted to regularize $\boldsymbol{R}_{i}$, and Eq. (4) can be rewritten as

$$
\begin{aligned}
\boldsymbol{A}_{i} & =\arg \min _{\boldsymbol{A}_{i}}\left\{\left\|\boldsymbol{Y}_{i}-\boldsymbol{D}_{i} \boldsymbol{A}_{i}\right\|_{F}^{2}+\lambda_{i}\left\|\boldsymbol{A}_{i}-\boldsymbol{B}_{i}\right\|_{1}\right\} \\
& =\arg \min _{\tilde{\boldsymbol{\alpha}}_{i}}\left\{\left\|\tilde{\boldsymbol{y}}_{i}-\tilde{\boldsymbol{D}}_{i} \tilde{\boldsymbol{\alpha}}_{i}\right\|_{2}^{2}+\lambda_{i}\left\|\tilde{\boldsymbol{\alpha}}_{i}-\tilde{\boldsymbol{\beta}}_{i}\right\|_{1}\right\}
\end{aligned}
$$

where $\tilde{\boldsymbol{y}}_{i}, \tilde{\boldsymbol{\alpha}}_{i}$, and $\tilde{\boldsymbol{\beta}}_{i}$ denote the vectorization of the matrix $\boldsymbol{Y}_{i}, \boldsymbol{A}_{i}$ and $\boldsymbol{B}_{i}$, respectively. Each column $\tilde{\boldsymbol{d}}_{j}$ of the matrix $\tilde{\boldsymbol{D}}_{i}=\left[\tilde{\boldsymbol{d}}_{1}, \tilde{\boldsymbol{d}}_{2}, \ldots, \tilde{\boldsymbol{d}}_{J}\right]$ denotes the vectorization of the rank-one matrix, where $J$ denotes the number of dictionary atoms. 


\subsection{Algorithm of GSRC-ENSS}

For fixed $\tilde{\boldsymbol{\beta}}_{i}$ and $\lambda_{i}$, it can be seen that Eq. (16) is convex and can be solved efficiently by using some iterative thresholding algorithms. We adopt the surrogate algorithm in 56 to solve Eq. (16). In the $t+1$-iteration, the proposed shrinkage operator can be calculated as

$$
\tilde{\boldsymbol{\alpha}}_{i}^{t+1}=\boldsymbol{S}_{\lambda_{i}}\left(\tilde{\boldsymbol{D}}_{i}^{-1} \hat{\tilde{\boldsymbol{x}}}_{i}^{t}-\tilde{\boldsymbol{\beta}}_{i}\right)+\tilde{\boldsymbol{\beta}}_{i}
$$

where $\boldsymbol{S}_{\lambda_{i}}(\cdot)$ is the soft-thresholding operator, $\hat{\tilde{\boldsymbol{x}}}_{i}$ represents the vectorization of the $i$-th reconstructed group $\hat{\boldsymbol{X}}_{i}$. In fact, according to Eq. (17), it can be seen that these two NSS priors can be better integrated into this surrogate algorithm. The above shrinkage operator follows the standard surrogate algorithm, and more details can be seen in [56.

The parameter $\lambda_{i}$ for each group that balances the fidelity term and the regularization term should be adaptively determined for better denoising performance. Inspired by [57, the regularization parameter $\lambda_{i}$ of each group $\boldsymbol{Y}_{i}$ is set as

$$
\lambda_{i}=\frac{c * 2 \sqrt{2} \sigma^{2}}{\sigma_{i}}
$$

where $\sigma_{i}$ denotes the estimated variance of each group sparsity residual $\boldsymbol{R}_{i}$, and $c$ is a small constant.

After obtaining the solution $\boldsymbol{A}_{i}$ in Eq. (17), the clean group $\boldsymbol{X}_{i}$ can be reconstructed as $\hat{\boldsymbol{X}}_{i}=\boldsymbol{D}_{i} \boldsymbol{A}_{i}$. Then the clean image $\hat{\boldsymbol{X}}$ can be reconstructed by aggregating all the groups $\left\{\hat{\boldsymbol{X}}_{i}\right\}$.

Besides, we could execute the above denoising procedures for better results after several iterations. In the $t$-th iteration, the iterative regularization strategy [58] is used to update the estimation of noise variance. Then the standard divation of noise in $t$-th iteration is adjusted as

$$
\sigma^{t+1}=\gamma * \sqrt{\left(\sigma^{2}-\left\|\boldsymbol{Y}-\hat{\boldsymbol{X}}^{t}\right\|_{2}^{2}\right)}
$$

where $\gamma$ is a constant. 
According to the above analysis, it can be seen that the proposed GSRCENSS employs the group sparsity residual with external NSS prior for image denoising. The proposed denoising procedure is summarized in Table 1 .

Table 1: Image denoising via GSRC-ENSS

Input: Noisy image $\boldsymbol{Y}$ and Group-based GMM learning model.

Initialization: $\hat{\boldsymbol{X}}=\boldsymbol{Y}, c, m, d, W, K, \sigma, \gamma, \rho$;

For $t=1,2, \ldots$, Iter do

Iterative regularization $\boldsymbol{Y}^{t+1}=\hat{\boldsymbol{X}}^{t}+\rho\left(\boldsymbol{Y}-\hat{\boldsymbol{X}}^{t}\right)$;

Re-estimate $\sigma^{t+1}$ computing by Eq. 19p;

For each patch $\boldsymbol{y}_{i}$ in $\boldsymbol{Y}$ do

Find a group $\boldsymbol{Y}_{i}^{t+1}$ for each patch $\boldsymbol{y}_{i}$.

The best Gaussian component is selected by Eq. (13).

Constructing dictionary $\boldsymbol{U}_{k}$ by Eq. (14).

Update $\boldsymbol{B}_{i}{ }^{t+1}$ computing by $\boldsymbol{B}_{i}=\boldsymbol{U}_{k}{ }^{-1} \boldsymbol{Y}_{i}$.

Constructing dictionary $\boldsymbol{D}_{i}{ }^{t+1}$ by Eq. 15.

Update $\lambda_{i}{ }^{t+1}$ computing by Eq. (18).

Update $\boldsymbol{A}_{i}{ }^{t+1}$ computing by Eq. 17).

Get the estimation $\boldsymbol{X}_{i}{ }^{t+1}=\boldsymbol{D}_{i}{ }^{t+1} \boldsymbol{A}_{i}{ }^{t+1}$.

\section{End for}

Aggregate $\boldsymbol{X}_{i}{ }^{t+1}$ to form the recovered image $\hat{\boldsymbol{X}}^{t+1}$.

\section{End for}

Output: $\hat{X}^{t+1}$.

\section{Discussion}

In this section, we provide detailed discussion about the main difference among the proposed GSRC-ENSS method, the NCSR method 25, a majority of NSS prior-based denoising methods and the popular methods combining external and internal priors. 
- The main difference between NCSR [25] and the proposed GSRC-ENSS method is that NCSR first obtained some good estimates of the sparse coding coefficients of the original image by the principle of NLM [1] and then centralized the sparse coding coefficients of the observed image to those estimates to improve the performance of denoising. However, since NLM depends on the weighted graph [59], it is unavoidable that the weighted manner leads to disturbance and inaccuracy [60. It is worth mentioning that the proposed GSRC-ENSS method does not involve in the weighted graph. In addition, NCSR is actually a patch-based sparse coding method, which usually ignores the relationship among similar patches 13, 23, 50, 53.

- NSS prior has shown great success in image denoising. A majority of existing denoising methods only exploit the NSS prior of noisy image [12, 13, 14, 23, 24, 25, 27, 30, 37, and few methods use the NSS prior from natural images [11. Different from the most existing NSS prior-based denoising methods, in this work we consider two kinds of NSS prior, i.e., NSS priors of noisy input image and natural images.

- In recent years, several works about combining internal and external priors for image denoising were proposed [39, 40, 41, 42, 61, 62, 63. For example, Mosseri et al. 39 developed a Patch-SNR measure to decide whether a noisy patch is denoised patch using internal and external priors. Luo et al. 42 proposed a prior learning algorithm that combines the internal and external priors from a Bayesian hyper-prior perspective. However, different from the most existing methods, which are only combining external patch and internal patch priors to a new hybrid prior [39, 40, 41, 42, 61] or using external patch prior guided internal prior [62, 63, the proposed GSRC-ENSS method exploits external NSS prior and internal NSS prior to image denoising. 


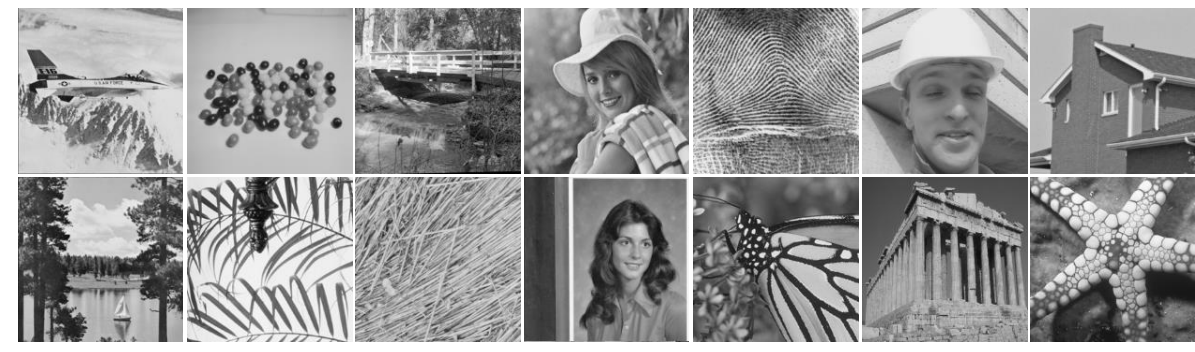

Figure 3: All test images. Top: Airplane, J.Bean, Bridge, elaine, F.print, foreman, House; bottom: Lake, Leaves, straw, Miss, Monarch, Parthenon, starfish.

\section{Experimental Results}

In this section, we validate the performance of the proposed GSRC-ENSS and compare it with several state-of-the-art denoising methods, including BM3D 12], EPLL [7, NCSR [25], GID 64, LINC 9], PGPD [11] and aGMM [42, where EPLL, LINC, PGPD and aGMM are based on GMM learning model and aGMM exploits external and internal priors. We evaluate the competing methods on 14 typical natural images, whose scenes are displayed in Fig. 3. Zero mean additive white Gaussian noises are added to those test images to generate the noisy observations. To evaluate the quality of denoised image, both PSNR and SSIM 65 metrics are used. The training groups used in our experiments were sampled based on NSS scheme from the Kodak photoCD dataset ${ }^{3}$, which includes 24 natural images. The detailed setting of parameters is shown in Table 2 including the number of Gaussian components $K^{4}$ search window $W$, the number of similar patches in a group $m$, patch size $d$ and involved parameters $c, \gamma, \rho$. The source code of the proposed method can be download at

\footnotetext{
${ }^{3}$ http://r0k.us/graphics/kodak/.

${ }^{4}$ Due to the operation of mean substraction, the possible patterns and variables of each group are reduced, and thus, the number of Gaussian component is not necessarily large. For the parameter $K$, the number of GMM components, we referred to a most recent work [11, and experimental results also shown that $K=64$ is the best choice under the limitation of testing environment.
} 
https://drive.google.com/open?id=0B0wKhHwcknCjWGx2LUdONnZibmM.

Table 2: Parameter settings

\begin{tabular}{|c|c|c|c|c|c|c|c|}
\hline & \multicolumn{3}{|c|}{ GMM Learning Stage } & \multicolumn{3}{|c|}{ Denoising Stage } \\
\hline Noise level & $K$ & $W$ & $d$ & $m$ & $c$ & $\rho$ & $\gamma$ \\
\hline$\sigma \leq 10$ & 64 & 50 & 6 & 80 & 0.14 & 0.19 & 1.08 \\
\hline $10<\sigma \leq 20$ & 64 & 50 & 6 & 80 & 0.13 & 0.20 & 1.05 \\
\hline $20<\sigma \leq 30$ & 64 & 50 & 7 & 90 & 0.12 & 0.21 & 1.05 \\
\hline $30<\sigma \leq 40$ & 64 & 50 & 8 & 100 & 0.11 & 0.22 & 1.05 \\
\hline $40<\sigma \leq 50$ & 64 & 50 & 8 & 100 & 0.10 & 0.23 & 1.05 \\
\hline $50<\sigma \leq 75$ & 64 & 50 & 9 & 120 & 0.09 & 0.24 & 1.00 \\
\hline $75<\sigma \leq 100$ & 64 & 50 & 9 & 120 & 0.08 & 0.25 & 1.00 \\
\hline
\end{tabular}

We present the denoising results on six noise levels, i.e., $\sigma=20,30,40,50,75$ and 100. The PSNR and SSIM results under these noise levels for all competing denoising methods are reported in Table 3 and Table 4 respectively (the highest PSNR and SSIM values are marked in bold). In a majority of cases, it can be easily found that the proposed GSRC-ENSS achieves better PSNR and SSIM results than other competing methods. In terms of PSNR, the proposed GSRCENSS achieves $0.21 \mathrm{~dB}, 0.56 \mathrm{~dB}, 0.29 \mathrm{~dB}, 1.29 \mathrm{~dB}, 0.32 \mathrm{~dB}, 0.13 \mathrm{~dB}$ and $0.39 \mathrm{~dB}$ improvement on average over the BM3D, EPLL, NCSR, GID, LINC, PGPD and aGMM, respectively. This clearly demonstrates the effectiveness of the proposed GSRC-ENSS.

Considering that human subjects are judging of the image quality at last, the visual quality of denoised images is also critical to evaluate a denoising algorithm. The visual comparisons of competing denoising methods at noise level 40, 50, 75 and 100 are shown in Fig. 4, Fig. 5, Fig. 6] and Fig. 7, respectively. It can be seen that BM3D and LINC are resulting in over-smooth phenomena, while EPLL, NCSR, GID, PGPD and aGMM are likely to generate some undesirable ringing artifacts. In contrast, the proposed GSRC-ENSS can not only recover sharp edges, fine details and suppress undesirable ringing artifacts, but 

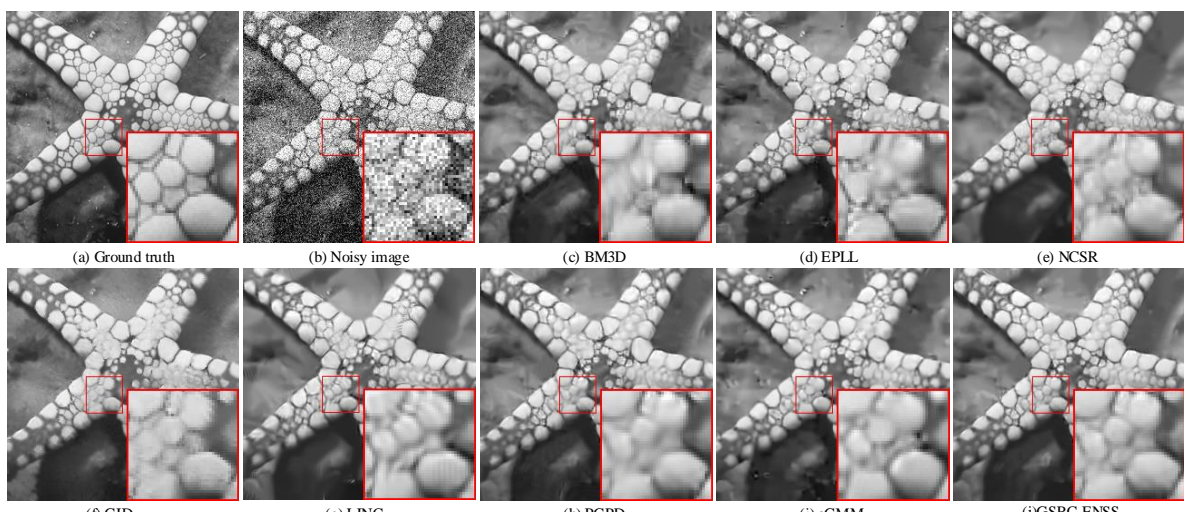

Figure 4: Denoising results on image starfish by different methods (noise level $\sigma=40$ ). (a) Original image; (b) Noisy image; (c) BM3D [12] $(\mathrm{PSNR}=26.06 \mathrm{~dB}, \mathrm{SSIM}=0.7828)$; (d) EPLL 7] $(\mathrm{PSNR}=26.12 \mathrm{~dB}, \mathrm{SSIM}=0.7802) ;(\mathrm{e}) \mathrm{NCSR}$ 25] $(\mathrm{PSNR}=26.17 \mathrm{~dB}, \mathrm{SSIM}=0.7812)$; (f) GID 64] $(\mathrm{PSNR}=25.39 \mathrm{~dB}, \mathrm{SSIM}=0.7565) ;(\mathrm{g}) \mathrm{LINC}$ 9] $(\mathrm{PSNR}=26.07 \mathrm{~dB}, \mathrm{SSIM}=0.7758) ;(\mathrm{h}) \mathrm{PG}-$ $\mathrm{PD}$ [1] $(\mathrm{PSNR}=26.21 \mathrm{~dB}, \mathrm{SSIM}=0.7855)$; (i) $\mathrm{aGMM}$ 42] $(\mathrm{PSNR}=26.16 \mathrm{~dB}, \mathrm{SSIM}=0.7824)$;

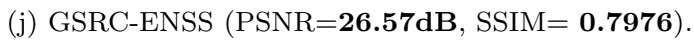

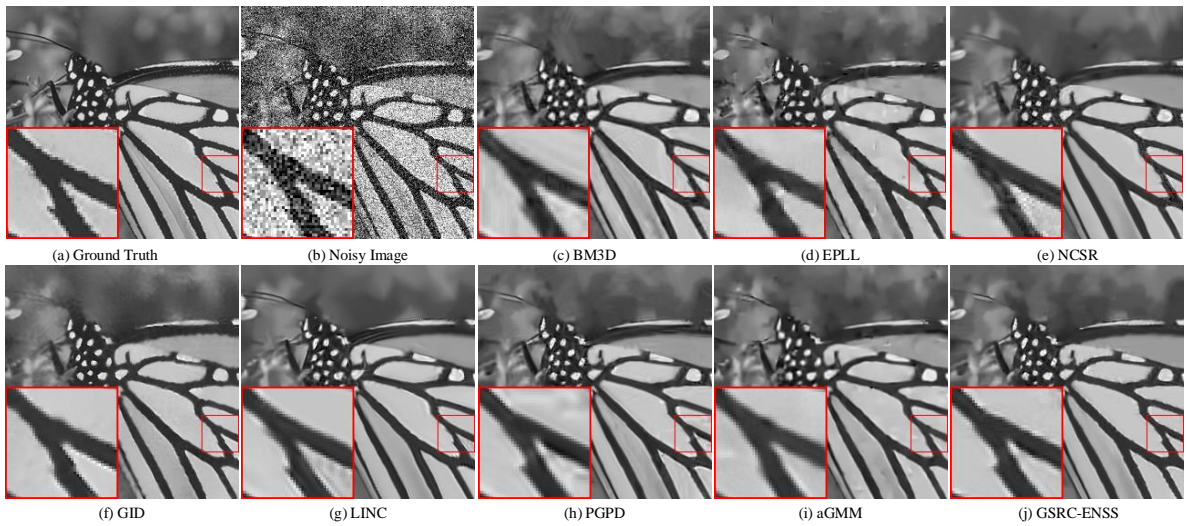

Figure 5: Denoising results on image Monarch by different methods (noise level $\sigma=50$ ). (a) Original image; (b) Noisy image; (c) BM3D [12] (PSNR=25.82dB, SSIM=0.8200); (d) EPLL 7] $(\mathrm{PSNR}=25.78 \mathrm{~dB}, \mathrm{SSIM}=0.8124) ;(\mathrm{e}) \mathrm{NCSR}$ 25] $(\mathrm{PSNR}=25.73 \mathrm{~dB}, \mathrm{SSIM}=0.8252)$; (f) GID [64] (PSNR=25.28dB, SSIM=0.8022); (g) LINC 9] $(\mathrm{PSNR}=25.88 \mathrm{~dB}, \mathrm{SSIM}=\mathbf{0 . 8 3 1 4})$; (h) PGPD [1] $(\mathrm{PSNR}=26.00 \mathrm{~dB}, \mathrm{SSIM}=0.8269)$; (i) $\mathrm{aGMM}$ 42] $(\mathrm{PSNR}=25.82 \mathrm{~dB}, \mathrm{~S}-$ $\mathrm{SIM}=0.8164)$; (j) GSRC-ENSS $(\mathrm{PSNR}=\mathbf{2 6 . 1 8 d B}, \mathrm{SSIM}=0.8269)$. 
Table 3: PSNR (dB) Comparison of BM3D [12, EPLL [7, NCSR 25], GID [64], LINC 9], PGPD [1], aGMM 42] and GSRC-ENSS.

\begin{tabular}{|c|c|c|c|c|c|c|c|c|c|c|c|c|c|c|c|c|}
\hline & \multicolumn{8}{|c|}{$\sigma=20$} & \multicolumn{8}{|c|}{$\sigma=30$} \\
\hline & BM3D & EPLL & NCSR & GID & LINC & PGPD & aGMM & $\begin{array}{c}\text { GSRC- } \\
\text { ENSS }\end{array}$ & ВM3D & EPLL & NCSR & GID I & LINC & PGPD & aGMM & $\begin{array}{c}\text { GSRC- } \\
\text { ENSS }\end{array}$ \\
\hline airplane & 30.59 & 30.60 & 30.50 & 29.62 & 30.57 & 30.79 & 30.54 & 30.81 & 28.49 & 28.54 & 28.34 & $27.54 \mid$ & 28.53 & 28.62 & 28.42 & 28.66 \\
\hline bridge & 27.27 & 27.50 & 27.28 & 26.39 & 27.31 & 27.31 & 27.20 & 27.27 & 25.47 & 25.67 & 25.44 & $24.80 \mid$ & 25.43 & 25.48 & 25.35 & 25.40 \\
\hline elaine & 32.51 & 32.16 & 32.39 & 31.23 & 32.58 & 32.43 & 32.62 & 32.41 & 30.52 & 30.15 & 30.25 & 28.98 & 30.39 & 30.47 & 30.59 & 30.42 \\
\hline F.print & 26.75 & 26.18 & 26.83 & 26.10 & 26.83 & 26.84 & 26.15 & 27.03 & 24.75 & 24.08 & 24.78 & 24.25 & 24.82 & 24.73 & 24.10 & 24.92 \\
\hline foreman & 34.54 & 33.67 & 34.42 & 33.08 & 34.76 & 34.44 & 34.20 & 34.45 & 32.75 & 31.70 & 32.61 & 30.92 & 32.93 & 32.83 & 32.31 & 32.96 \\
\hline House & 33.77 & 32.99 & 33.81 & 32.68 & 33.82 & 33.85 & 33.52 & 33.82 & 32.08 & 31.24 & 32.01 & $\mid 30.50$ & 32.26 & 32.24 & 31.79 & 32.34 \\
\hline J.Bean & 34.18 & 33.79 & 34.37 & 33.38 & 34.14 & 34.28 & 34.80 & 34.68 & 31.97 & 31.55 & 31.99 & $|30.74|$ & 31.82 & 31.99 & 32.50 & 32.39 \\
\hline Lake & 28.85 & 29.06 & 28.85 & 28.17 & 28.97 & 29.07 & 28.79 & 29.11 & 26.74 & 26.97 & 26.69 & 26.03 & 26.95 & 26.90 & 26.70 & 26.98 \\
\hline Leave & 30.09 & 29.40 & 30.34 & 29.91 & 30.24 & 30.46 & 30.05 & 30.54 & 27.81 & 27.19 & 28.04 & 27.59 & 27.99 & 27.99 & 27.53 & 28.15 \\
\hline Miss & 33.71 & 33.33 & 33.58 & 32.17 & 33.64 & 33.66 & 33.84 & 33.58 & 31.89 & 31.40 & 31.62 & $|30.10|$ & 31.75 & 31.88 & 32.04 & 31.78 \\
\hline Monarch & 30.35 & 30.49 & 30.52 & 29.75 & 30.64 & 30.68 & 30.31 & 30.81 & 28.36 & 28.36 & 28.38 & 27.68 & 28.74 & 28.49 & 28.27 & 28.69 \\
\hline Parthenon & 29.45 & 29.23 & 29.41 & 28.15 & 29.38 & 29.33 & 29.42 & 29.26 & 27.54 & 27.32 & 27.48 & 26.25 & 27.49 & 27.39 & 27.43 & 27.37 \\
\hline starfish & 29.67 & 29.58 & 29.85 & 29.05 & 29.58 & 29.84 & 29.74 & 30.05 & 27.65 & 27.52 & 27.69 & 26.90 & 27.52 & 27.67 & 27.61 & 28.02 \\
\hline straw & 27.08 & 26.93 & 27.38 & 26.60 & 27.42 & 27.18 & 26.66 & 27.48 & 24.91 & 24.76 & 25.08 & 24.66 & 25.51 & 24.83 & 24.44 & 25.27 \\
\hline \multirow[t]{3}{*}{ Average } & 30.63 & 30.35 & 30.68 & 29.73 & 30.71 & 30.72 & 30.56 & 30.81 & 28.64 & 28.32 & 28.60 & $|27.64|$ & 28.72 & 28.68 & 28.50 & 28.81 \\
\hline & \multicolumn{8}{|c|}{$\sigma=40$} & \multicolumn{8}{|c|}{$\sigma=50$} \\
\hline & ВM3D & EPLL & NCSR & GID & LINC 1 & PGPD & aGMM & $\begin{array}{c}\text { GSRC- } \\
\text { ENSS }\end{array}$ & ВM3D & EPLL & NCSR & GID I & LINC & PGPD & aGMM & $\begin{array}{l}\text { GSRC- } \\
\text { ENSS }\end{array}$ \\
\hline airplane & 26.88 & 27.08 & 26.78 & 26.17 & 27.08 & 27.12 & 26.95 & 27.18 & 25.76 & 25.96 & 25.63 & 25.10 & 26.04 & 25.98 & 25.83 & 25.99 \\
\hline bridge & 24.33 & 24.51 & 24.21 & 23.73 & 24.10 & 24.38 & 24.25 & 24.31 & 23.58 & 23.69 & 23.41 & 22.78 & 23.18 & 23.60 & 23.50 & 23.55 \\
\hline elaine & 28.95 & 28.73 & 28.91 & 27.67 & 28.96 & 29.08 & 29.10 & 28.95 & 27.96 & 27.63 & 27.68 & 26.54 & 27.73 & 27.90 & 27.93 & 27.78 \\
\hline F.print & 22.88 & 22.66 & 23.29 & 23.00 & 23.42 & 23.33 & 22.77 & 23.50 & 22.59 & 21.55 & 22.29 & 21.89 & 22.19 & 22.36 & 21.74 & 22.50 \\
\hline foreman & 31.29 & 30.28 & 31.52 & 29.61 & 31.31 & 31.55 & 30.95 & 31.77 & 30.56 & 29.20 & 30.41 & 28.64 & 30.33 & 30.45 & 29.80 & 30.57 \\
\hline House & 30.65 & 29.89 & 30.79 & 29.02 & 31.00 & 31.02 & 30.40 & 31.12 & 29.69 & 28.79 & 29.61 & 27.76 & 29.87 & 29.93 & 29.28 & 30.14 \\
\hline J.Bean & 30.22 & 29.96 & 30.49 & 29.53 & 30.24 & 30.39 & 30.79 & 30.74 & 29.26 & 28.73 & 29.24 & 28.43 & 29.01 & 29.20 & 29.46 & 29.47 \\
\hline Lake & 25.21 & 25.58 & 25.21 & 24.61 & 25.51 & 25.51 & 25.39 & 25.56 & 24.29 & 24.56 & 24.15 & 23.68 & 24.30 & 24.49 & 24.39 & 24.51 \\
\hline Leave & 25.69 & 25.62 & 26.20 & 25.82 & 26.31 & 26.29 & 25.76 & 26.43 & 24.68 & 24.39 & 24.94 & 24.41 & 25.11 & 25.03 & 24.42 & 25.23 \\
\hline Miss & 30.50 & 29.93 & 30.46 & 28.89 & 30.29 & 30.54 & 30.65 & 30.49 & 29.48 & 28.76 & 29.31 & 27.80 & 29.22 & 29.45 & 29.42 & 29.31 \\
\hline Monarch & 26.72 & 26.89 & 26.81 & 26.32 & 27.14 & 27.02 & 26.87 & 27.22 & 25.82 & 25.78 & 25.73 & 25.28 & 25.88 & 26.00 & 25.82 & 26.18 \\
\hline Parthenon & 26.15 & 25.95 & 26.03 & 24.88 & 26.08 & 26.13 & 26.07 & 26.14 & 25.26 & 25.02 & 25.12 & 24.06 & 25.15 & 25.22 & 25.06 & 25.26 \\
\hline starfish & 26.06 & 26.12 & 26.17 & 25.39 & 26.07 & 26.21 & 26.16 & 26.57 & 25.04 & 25.05 & 25.06 & 24.07 & 24.81 & 25.11 & 25.09 & 25.45 \\
\hline straw & 23.16 & 23.19 & 23.62 & 23.25 & 23.91 & 23.39 & 22.94 & 23.82 & 22.41 & 22.02 & 22.48 & 22.02 & 22.34 & 22.30 & 21.82 & 22.64 \\
\hline \multirow[t]{3}{*}{ Average } & 27.05 & 26.89 & 27.18 & 26.28 & 27.25 & 27.28 & 27.07 & 27.41 & 26.16 & 25.79 & 26.07 & 25.18 & 26.08 & 26.22 & 25.97 & 26.33 \\
\hline & \multicolumn{8}{|c|}{$\sigma=75$} & \multicolumn{8}{|c|}{$\sigma=100$} \\
\hline & BM3D & EPLL & NCSR & GID & LINC 1 & PGPD & aGMM & $\begin{array}{c}\text { GSRC- } \\
\text { ENSS }\end{array}$ & BM3D & EPLL & NCSR & GID I & LINC & PGPD & aGMM & $\begin{array}{l}\text { GSRC- } \\
\text { ENSS }\end{array}$ \\
\hline airplane & 23.99 & 24.03 & 23.76 & 22.91 & 23.71 & 24.15 & 23.95 & 24.13 & 22.89 & 22.78 & 22.60 & 21.82 & 22.42 & 23.03 & 22.67 & 22.97 \\
\hline bridge & 22.33 & 22.30 & 22.00 & 21.30 & 21.71 & 22.32 & 22.26 & 22.34 & 21.44 & 21.36 & 21.06 & 20.46 & 20.61 & 21.42 & 21.35 & 21.33 \\
\hline elaine & 25.93 & 25.60 & 25.34 & 24.54 & 25.42 & 25.78 & 25.77 & 25.72 & 24.48 & 24.16 & 23.77 & 23.21 & 23.92 & 24.34 & 24.30 & 24.24 \\
\hline F.print & 21.09 & 19.34 & 20.59 & 19.47 & 20.14 & 20.70 & 19.56 & 20.88 & 20.03 & 17.65 & 19.51 & 17.66 & 18.87 & 19.57 & 17.56 & 19.68 \\
\hline foreman & 28.07 & 27.24 & 28.18 & 26.71 & 28.11 & 28.39 & 27.67 & 28.76 & 26.51 & 25.91 & 26.55 & 25.33 & 26.55 & 26.81 & 26.20 & 27.27 \\
\hline House & 27.51 & 26.70 & 27.16 & 25.23 & 27.56 & 27.81 & 27.11 & 28.21 & 25.87 & 25.21 & 25.49 & 22.38 & 26.11 & 26.17 & 25.55 & 26.44 \\
\hline J.Bean & 27.22 & 26.57 & 27.15 & 26.26 & 26.62 & 27.07 & 27.09 & 27.43 & 25.80 & 25.16 & 25.61 & 24.37 & 24.88 & 25.66 & 25.58 & 26.07 \\
\hline Lake & 22.63 & 22.77 & 22.48 & 21.82 & 22.45 & 22.76 & 22.68 & 22.72 & 21.56 & 21.60 & 21.37 & 20.69 & 20.99 & 21.66 & 21.55 & 21.63 \\
\hline Leave & 22.49 & 22.03 & 22.60 & 21.28 & 22.45 & 22.61 & 21.96 & 22.89 & 20.90 & 20.26 & 20.84 & 19.13 & 20.49 & 20.95 & 20.29 & 21.44 \\
\hline Miss & 27.34 & 26.68 & 27.03 & 25.47 & 26.70 & 27.51 & 27.25 & 27.48 & 25.67 & 25.23 & 25.24 & 23.73 & 25.28 & 25.94 & 25.71 & 26.13 \\
\hline Monarch & 23.91 & 23.73 & 23.67 & 22.77 & 23.91 & 24.00 & 23.85 & 24.21 & 22.52 & 22.24 & 22.10 & 20.73 & 22.13 & 22.56 & 22.42 & 22.83 \\
\hline Parthenon & 23.61 & 23.45 & 23.35 & 22.52 & 23.37 & 23.68 & 23.38 & 23.85 & 22.57 & 22.39 & 22.39 & 21.50 & 22.21 & 22.70 & 22.42 & 22.86 \\
\hline starfish & 23.28 & 23.17 & 23.18 & 21.99 & 22.74 & 23.23 & 23.22 & 23.45 & 22.10 & 21.92 & 21.91 & 20.84 & 21.10 & 22.08 & 21.95 & 22.10 \\
\hline straw & 20.72 & 20.09 & 20.62 & 19.70 & 19.85 & 20.58 & 19.90 & 20.81 & 19.59 & 19.03 & 19.39 & 18.38 & 18.75 & 19.57 & 18.81 & 19.49 \\
\hline Average & 24.29 & 23.84 & 24.08 & 23.00 & 23.92 & 24.33 & 23.97 & 24.49 & 22.99 & 22.49 & 22.70 & $21.45 \mid$ & 22.45 & 23.03 & 22.60 & 23.18 \\
\hline
\end{tabular}


Table 4: SSIM Comparison of BM3D [12, EPLL 7], NCSR [25, GID 64], LINC 9], PGPD 11, aGMM 42 and GSRC-ENSS.

\begin{tabular}{|c|c|c|c|c|c|c|c|c|c|c|c|c|c|c|c|c|}
\hline & \multicolumn{8}{|c|}{$\sigma=20$} & \multicolumn{8}{|c|}{$\sigma=30$} \\
\hline & BM3D & EPLL & NCSR & GID & LINC & PGPD & aGMM & $\begin{array}{c}\text { GSRC- } \\
\text { ENSS }\end{array}$ & BM3D & EPLL & NCSR & GID & LINC & PGPD & aGMM & $\begin{array}{l}\text { GSRC- } \\
\text { ENSS }\end{array}$ \\
\hline airplane & 0.9006 & 0.9017 & 0.9016 & 0.8837 & 0.8988 & 0.8986 & 0.9018 & 0.9022 & 0.8631 & 0.8628 & 0.8660 & 0.8449 & 0.8632 & 0.8641 & 0.8647 & 0.8673 \\
\hline elaine & 0.8900 & 0.8813 & 0.8880 & 0.8645 & 0.8927 & 0.8867 & 0.8914 & 0.8865 & 0.8530 & 0.8399 & 0.8498 & 0.8076 & 0.8517 & 0.8522 & 0.8542 & 0.8518 \\
\hline F.print & 0.9252 & 0.9212 & 0.9226 & 0.9034 & 0.9318 & 0.9219 & 0.9158 & 0.9255 & 0.8786 & 0.8680 & 0.8746 & 0.8520 & 0.8789 & 0.8699 & 0.8577 & 0.8760 \\
\hline J.Bean & 0.9582 & 0.9523 & 0.9632 & 0.9577 & 0.9615 & 0.9508 & 0.9633 & 0.9597 & 0.9357 & 0.9240 & 0.9435 & 0.9338 & 0.9449 & 0.9317 & 0.9413 & 0.9425 \\
\hline Lake & 0.8798 & 0.8826 & 0.8779 & 0.8543 & 0.8798 & 0.8803 & 0.8768 & 0.8809 & 0.8287 & 0.8318 & 0.8271 & 0.8013 & 0.8276 & 0.8294 & 0.8242 & 0.8325 \\
\hline Leave & 0.9534 & 0.9480 & 0.95550 & 0.9493 & 0.9561 & 0.9562 & 0.9559 & 0.9576 & 0.9378 & 0.9197 & 0.9311 & 0.9248 & 0.9339 & 0.9300 & 0.9273 & 0.9344 \\
\hline Miss & 0.9015 & 0.8980 & 0.9001 & 0.8777 & 0.9028 & 0.8969 & 0.9038 & 0.8999 & 0.8715 & 0.8648 & 0.8715 & 0.8408 & 0.8747 & 0.8706 & 0.8762 & 0.8748 \\
\hline Monarch & 0.9179 & 0.9166 & 0.9192 & 0.9027 & 0.9262 & 0.9187 & 0.9202 & 0.9198 & 0.8822 & 0.8789 & 0.8829 & 0.8628 & 0.8970 & 0.8853 & 0.8831 & 0.8857 \\
\hline \multirow{2}{*}{ Average } & \multicolumn{8}{|c|}{$\sigma=40$} & \multicolumn{8}{|c|}{$\sigma=50$} \\
\hline & BM3D & EPLL & NCSR & GID & LINC & PGPD & aGMM & $\begin{array}{c}\text { GSRC- } \\
\text { ENSS }\end{array}$ & BM3D & EPLL & NCSR & GID & LINC & PGPD & aGMM & $\begin{array}{l}\text { GSRC- } \\
\text { ENSS }\end{array}$ \\
\hline airplane & 0.8277 & 0.8264 & 0.8330 & 0.8120 & 0.8313 & 0.8338 & 0.8305 & 0.8384 & 0.8044 & 0.7922 & 0.8066 & 0.7795 & 0.8021 & 0.8053 & 0.7990 & 0.8094 \\
\hline bridge & 0.6365 & 0.6582 & 0.6187 & 0.5994 & 0.6027 & 0.6325 & 0.6225 & 0.6242 & 0.5834 & 0.6034 & 0.569 & 0.5353 & 0.5366 & 0.5824 & 0.5745 & 0.5748 \\
\hline elaine & 0.8180 & 0.8047 & 0.8258 & 0.7838 & 0.8244 & 0.8223 & 0.8209 & 0.8219 & 0.7971 & 0.7741 & 0.7976 & 0.7494 & 0.7945 & 0.7926 & 0.7890 & 0.7925 \\
\hline F.print & 0.8188 & 0.8117 & 0.8123 & 0.8072 & 0.8243 & 0.8212 & 0.8015 & 0.8286 & 0.7933 & 0.7528 & 0.7598 & 0.7581 & 0.7698 & 0.7760 & 0.7472 & 0.7831 \\
\hline foreman & 0.8565 & 0.8315 & 0.8723 & 0.8262 & 0.8675 & 0.8621 & 0.8515 & 0.8701 & 0.8445 & 0.8051 & 0.8559 & 0.8080 & 0.8534 & 0.8410 & 0.8270 & 0.8482 \\
\hline House & 0.8256 & 0.8089 & 0.8323 & 0.7979 & 0.8340 & 0.8302 & 0.8221 & 0.8355 & 0.8122 & 0.7845 & 0.8160 & 0.7718 & 0.8180 & 0.8125 & 0.8002 & 0.8202 \\
\hline J.Bean & 0.9122 & 0.8956 & 0.9296 & 0.9139 & 0.9248 & 0.9133 & 0.9170 & 0.9262 & 0.9006 & 0.8677 & 0.9134 & 0.8956 & 0.9085 & 0.8934 & 0.8911 & 0.9047 \\
\hline Lake & 0.7793 & 0.7871 & 0.7788 & 0.7541 & 0.7834 & 0.7859 & 0.7812 & 0.7907 & 0.7433 & 0.7477 & 0.7420 & 0.7121 & 0.7423 & 0.7483 & 0.7428 & 0.7517 \\
\hline \multirow{2}{*}{ Average } & \multicolumn{8}{|c|}{$\sigma=75$} & \multicolumn{8}{|c|}{$\sigma=100$} \\
\hline & BM3D & EPLL & NCSR & GID & LINC & PGPD & aGMM & $\begin{array}{c}\text { GSRC- } \\
\text { ENSS }\end{array}$ & BM3D & EPLL & NCSR & GID & LINC & PGPD & aGMM & $\begin{array}{c}\text { GSRC- } \\
\text { ENSS }\end{array}$ \\
\hline airplane & 0.7488 & 0.7168 & 0.7547 & 0.6754 & 0.7470 & 0.7484 & 0.7248 & 0.7553 & 0.7036 & 0.6523 & 0.7107 & 0.6393 & 0.6931 & 0.6940 & 0.6571 & 0.7038 \\
\hline bridge & 0.5086 & 0.5089 & 0.4820 & 0.4413 & 0.4391 & 0.5013 & 0.4939 & 0.5007 & 0.4585 & 0.4483 & 0.4259 & 0.3883 & 0.3797 & 0.4481 & 0.4361 & 0.4661 \\
\hline elaine & 0.7359 & 0.7062 & 0.7342 & 0.6762 & 0.7280 & 0.7303 & 0.7172 & 0.7353 & 0.6817 & 0.6459 & 0.6824 & 0.6139 & 0.6812 & 0.6721 & 0.6548 & 0.6801 \\
\hline F.print & 0.7102 & 0.5814 & 0.6574 & 0.5952 & 0.6224 & 0.6755 & 0.5705 & 0.6944 & 0.6381 & 0.3837 & 0.5814 & 0.4179 & 0.5165 & 0.5875 & 0.3460 & 0.6074 \\
\hline foreman & 0.7933 & 0.7467 & 0.8171 & 0.7556 & 0.8162 & 0.7965 & 0.7676 & 0.8096 & 0.7489 & 0.6949 & 0.7833 & 0.7050 & 0.7826 & 0.7452 & 0.7129 & 0.7656 \\
\hline House & 0.7645 & 0.7251 & 0.7749 & 0.7052 & 0.7850 & \begin{tabular}{|l|}
0.7709 \\
\end{tabular} & 0.7419 & 0.7877 & 0.7203 & 0.6695 & 0.7397 & 0.4735 & 0.7550 & 0.7195 & 0.6854 & 0.7401 \\
\hline J.Bean & 0.8573 & 0.8019 & 0.8792 & 0.8574 & 0.8669 & 0.8503 & 0.8243 & 0.8658 & 0.8181 & 0.7429 & 0.8472 & 0.7862 & 0.8339 & 0.7999 & 0.7628 & 0.8218 \\
\hline Lake & 0.6716 & 0.6644 & 0.6743 & 0.6366 & 0.6590 & 0.6760 & 0.6612 & 0.6787 & 0.6178 & 0.5977 & 0.6235 & 0.5728 & 0.5940 & 0.6171 & 0.5965 & 0.6224 \\
\hline Leave & 0.8072 & 0.7921 & 0.8234 & 0.7751 & 0.8247 & 0.8121 & 0.7867 & 0.8337 & 0.7482 & 0.7163 & 0.7622 & 0.6857 & 0.7499 & 0.7469 & 0.7106 & 0.7874 \\
\hline Miss & 0.7722 & 0.7422 & 0.7936 & 0.7353 & 0.7819 & 0.7757 & 0.7626 & 0.7867 & 0.7240 & 0.6901 & 0.7576 & 0.6607 & 0.7438 & 0.7236 & 0.7090 & 0.7465 \\
\hline Monarch & 0.8200 & 0.8124 & 0.8252 & 0.8022 & 0.8314 & 0.8269 & 0.8164 & 0.8269 & 0.7021 & 0.6771 & 0.7109 & 0.6361 & 0.7076 & 0.7029 & 0.6823 & 0.7874 \\
\hline Parthenon & 0.6202 & 0.5968 & 0.5980 & 0.5550 & 0.5929 & 0.6182 & 0.5888 & 0.6183 & 0.557 & 0.5397 & 0.5535 & 0.5052 & 0.5425 & 0.5638 & 0.5340 & 0.5635 \\
\hline starfish & 0.6670 & 0.6502 & 0.6685 & 0.6111 & 0.6416 & 0.6638 & 0.6525 & 0.6808 & 0.6053 & 0.5799 & 0.6062 & 0.5415 & 0.5635 & 0.6018 & 0.5813 & 0.6111 \\
\hline straw & 0.5462 & 0.4567 & 0.5291 & 0.4337 & 0.4143 & 0.5177 & 0.4150 & 0.5568 & 0.4224 & 0.3295 & 0.3722 & 0.3013 & 0.2687 & 0.4033 & 0.2819 & 0.3916 \\
\hline Average & 0.7113 & 0.6735 & 0.7108 & 0.6543 & 0.6922 & 0.7072 & 0.6752 & 0.7195 & 0.6533 & 0.5977 & 0.6541 & 0.5662 & 0.6294 & 0.6447 & 0.5965 & 0.6597 \\
\hline
\end{tabular}


also yield visually pleasant results. For example, GSRC-ENSS achieves the most visually pleasant restoration of the images starfish in Fig. 4 and House in Fig. 6 especially when one inspects the zoomed portions. Moreover, it can be seen that although PSNR results are slightly lower than BM3D and PGPD methods in Fig. 8 and Fig. 9. BM3D and PGPD methods generate obvious ringing artifacts. Note that BM3D only uses the NSS prior of noisy image and the NSS prior of natural images is used in PGPD. However, the proposed GSRC-ENSS can generate more details and less ringing artifacts. The reason for this is that two NSS priors (i.e., NSS priors of noisy image and natural images) are used in our GSRC model and the iterative shrinkage algorithm (Eq. (17)) can better combine these two NSS priors.

We also evaluate different denoising methods on two real images, including BM3D, PGPD and the proposed GSRC-ENSS methods. The visual comparisons of competing denoising methods are shown in Fig. 10 and Fig. 11. One can observe that the proposed GSRC-ENSS is able to preserve the sharp edges and small fine details more effective than BM3D and PGPD methods. The results show the feasibility of deploying our GSRC-ENSS method for some practical image denoising applications.

Moreover, to evaluate the computational cost of eight denoising methods, we compare the running time on all test images with different noise levels. All experiments are run under the Matlab2012b environment on a machine with Intel(R) Core(TM) i3-4150 with a 3.50GHz CPU and 4GB RAM, and Windows 10 operating system. The average run time(s) of computing methods on all test images is shown in Table 5. It's noted that BM3D and GID methods use parallel computing. Meanwhile, BM3D method is implemented with compiled $\mathrm{C}++$ mex-function, while the other methods are implemented in Matlab. It can be seen that the proposed GSRC-ENSS method often needs more time than other competing methods. Nonetheless, the proposed GSRC-ENSS delivers the best qualitative denoising results with finer details and less ringing artifacts, such as Figs. 4 - 11 .

To sump up, such experimental findings clearly demonstrate that the GSRC- 
Table 5: Average run time (s) on all test images with different methods.

\begin{tabular}{|c|c|c|c|c|c|c|c|c|}
\hline Methods & BM3D & EPLL & NCSR & GID & LINC & PGPD & aGMM & GSRC-ENSS \\
\hline Average Time (s) & 3 & 112 & 355 & 346 & 254 & 10 & 249 & 699 \\
\hline
\end{tabular}

320 large variations in edges/textures.
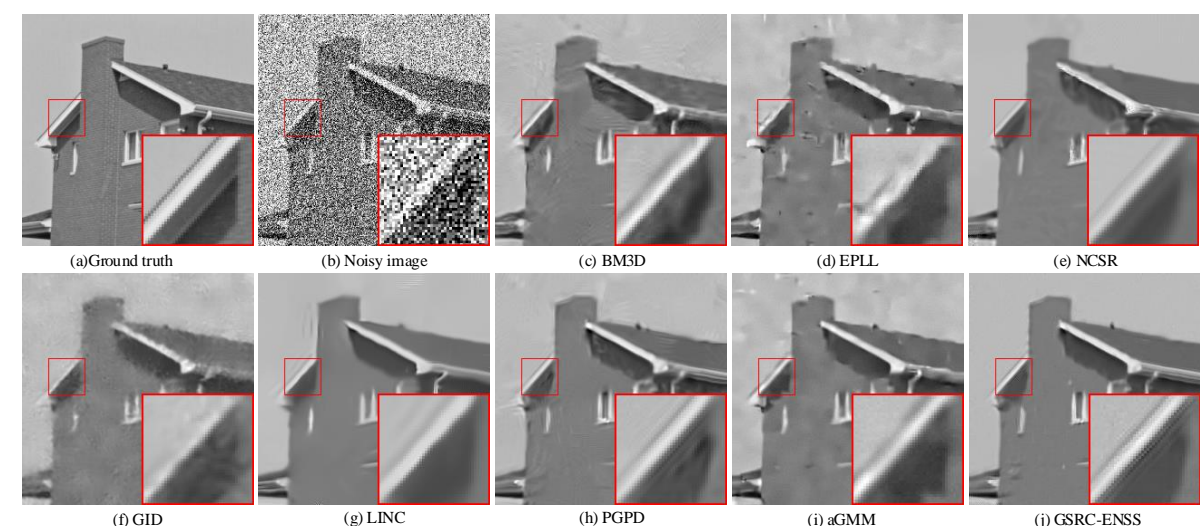

Figure 6: Denoising results on image House by different methods (noise level $\sigma=75$ ). (a) Original image; (b) Noisy image; (c) BM3D 12] (PSNR=27.51dB, SSIM=0.7645); (d) EPLL 7] $(\mathrm{PSNR}=26.70 \mathrm{~dB}, \mathrm{SSIM}=0.7251) ;(\mathrm{e}) \mathrm{NCSR}$ 25] $(\mathrm{PSNR}=27.16 \mathrm{~dB}, \mathrm{SSIM}=0.7749) ;$ (f) GID 64] $(\mathrm{PSNR}=25.23 \mathrm{~dB}, \mathrm{SSIM}=0.7052) ;(\mathrm{g}) \mathrm{LINC}$ 9] $(\mathrm{PSNR}=27.56 \mathrm{~dB}, \mathrm{SSIM}=0.7850) ;(\mathrm{h}) \mathrm{PG}-$ $\mathrm{PD}$ [1] $(\mathrm{PSNR}=27.81 \mathrm{~dB}, \mathrm{SSIM}=0.7709)$; (i) aGMM 42] $(\mathrm{PSNR}=27.11 \mathrm{~dB}, \mathrm{SSIM}=0.7419)$; (j) GSRC-ENSS $(P S N R=\mathbf{2 8 . 2 1 d B}, S S I M=0.7877)$.

\section{Conclusion}

In this paper, a novel denoising method based on group sparsity residual constraint with external NSS prior (GSRC-ENSS) was presented. Our approach differed from typical NSS based denoising methods, which only exploited the nonlocal self-similarity (NSS) prior of noisy image or natural images, two NSS priors (i.e., NSS priors of noisy image and natural images) were used for image denoising. To boost the performance of image denoising, we proposed the concept of group sparsity residual, and thus the problem of image denoising was 


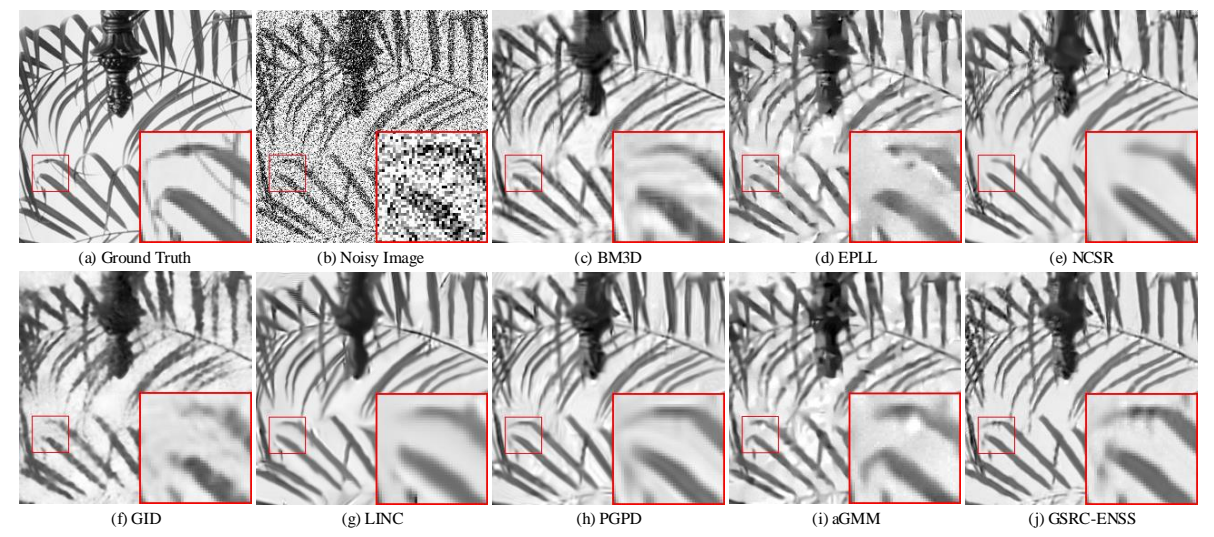

Figure 7: Denoising results on image Leaves by different methods (noise level $\sigma=100$ ). (a) Original image; (b) Noisy image; (c) BM3D [12] (PSNR=20.90dB, SSIM=0.7482); (d) EPLL 7] $(\mathrm{PSNR}=20.26 \mathrm{~dB}, \mathrm{SSIM}=0.7163) ;(\mathrm{e}) \mathrm{NCSR}$ 25] $(\mathrm{PSNR}=20.84 \mathrm{~dB}, \mathrm{SSIM}=0.7622)$; (f) GID 64] $(\mathrm{PSNR}=19.13 \mathrm{~dB}, \mathrm{SSIM}=0.6857)$; (g) LINC 9] $(\mathrm{PSNR}=20.49 \mathrm{~dB}, \mathrm{SSIM}=0.7499) ;(\mathrm{h}) \mathrm{PG}-$ $\mathrm{PD}$ [1] $(\mathrm{PSNR}=20.95 \mathrm{~dB}, \mathrm{SSIM}=0.7469)$; (i) $\mathrm{aGMM}$ 42] $(\mathrm{PSNR}=20.29 \mathrm{~dB}, \mathrm{SSIM}=0.7106)$; (j) GSRC-ENSS $(P S N R=\mathbf{2 1 . 4 4 d B}, \operatorname{SSIM}=\mathbf{0 . 7 8 7 4})$.

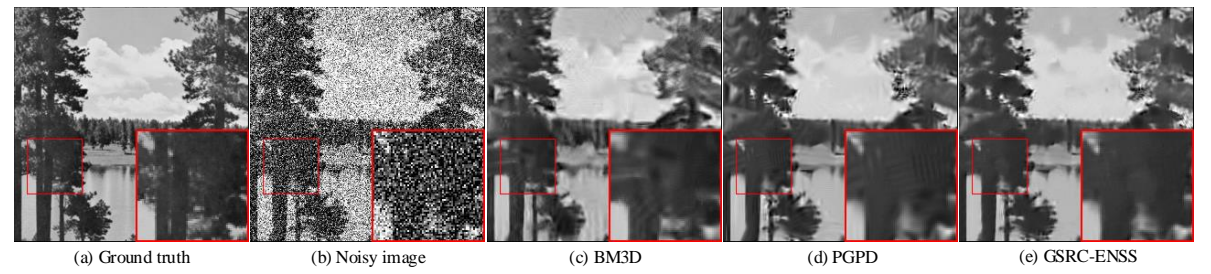

Figure 8: Denoising results on image Lake by different methods (noise level $\sigma=75$ ). (a) Original image; (b) Noisy image; (c) BM3D 12 (PSNR=22.63dB, SSIM=0.6716); (d) PGPD 11. $(\mathrm{PSNR}=\mathbf{2 2 . 7 6 d B}, \mathrm{SSIM}=0.6760)$; (e) GSRC-ENSS $(\mathrm{PSNR}=22.72 \mathrm{~dB}, \mathrm{SSIM}=\mathbf{0 . 6 7 8 7})$. 


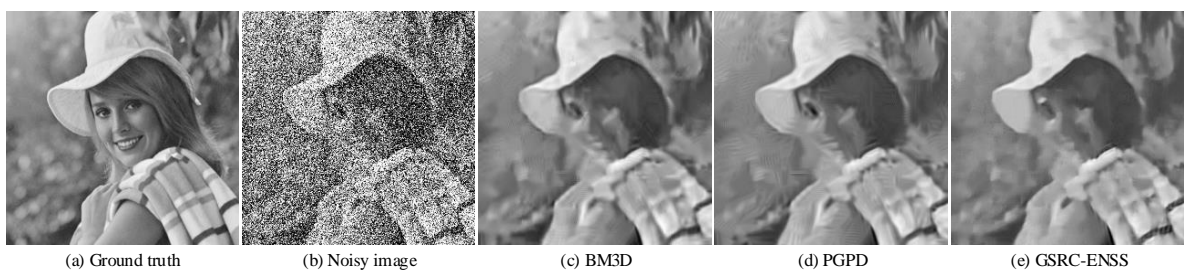

Figure 9: Denoising results on image elaine by different methods (noise level $\sigma=100$ ). (a) Original image; (b) Noisy image; (c) BM3D 12$](P S N R=\mathbf{2 4 . 4 8 d B}, \mathrm{SSIM=0.6817})$; (d) PGPD 11. $(\mathrm{PSNR}=24.34 \mathrm{~dB}, \mathrm{SSIM}=0.6721)$; (e) GSRC-ENSS $(\mathrm{PSNR}=24.24 \mathrm{~dB}, \mathrm{SSIM}=0.6801)$.

transformed into reducing the group sparsity residual. To this end, we achieved a good estimation of the group sparse coefficients of the original image by the NSS prior of natural images based GMM learning, and the group sparse coefficients of noisy image were exploited to approximate the estimation. To combine these two NSS priors better, we presented an iterative shrinkage algorithm to solve the GSRC-ENSS model. Experimental results have demonstrated that GSRC-ENSS can not only leads to visible improvements over many state-ofthe-art methods, but also preserves much better the image local structures and suppresses the ringing artifacts.

\section{References}

[1] A. Buades, B. Coll, J.-M. Morel, A non-local algorithm for image denoising, in: Computer Vision and Pattern Recognition, 2005. CVPR 2005. IEEE Computer Society Conference on, Vol. 2, IEEE, 2005, pp. 60-65.

[2] L. I. Rudin, S. Osher, E. Fatemi, Nonlinear total variation based noise removal algorithms, Physica D: Nonlinear Phenomena 60 (1-4) (1992) 259268.

[3] A. De Decker, J. A. Lee, M. Verleysen, A principled approach to image denoising with similarity kernels involving patches, Neurocomputing 73 (7) (2010) 1199-1209. 


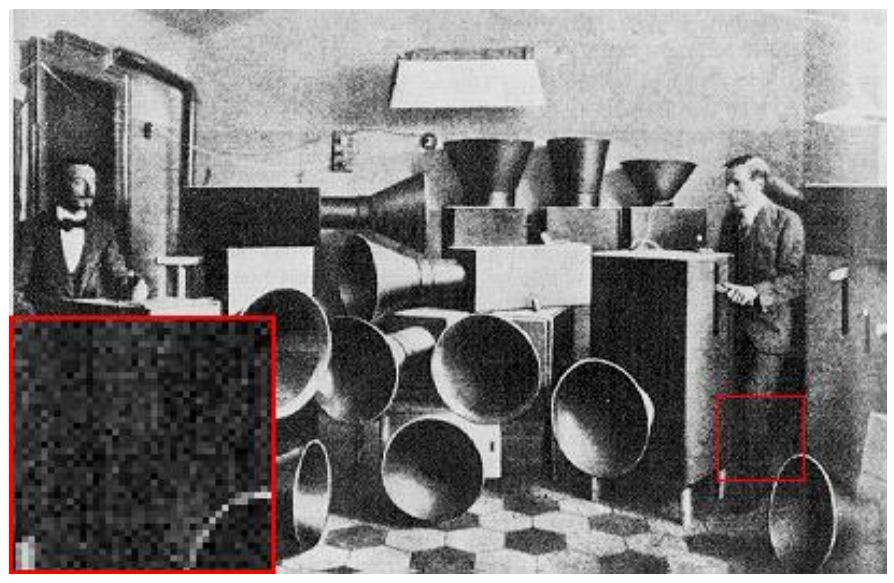

(a) Noisy image

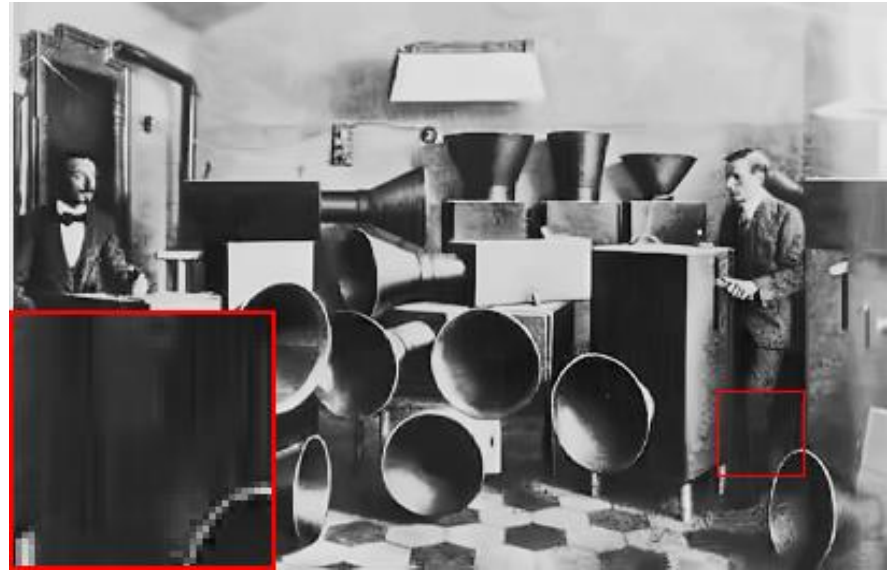

(c) PGPD

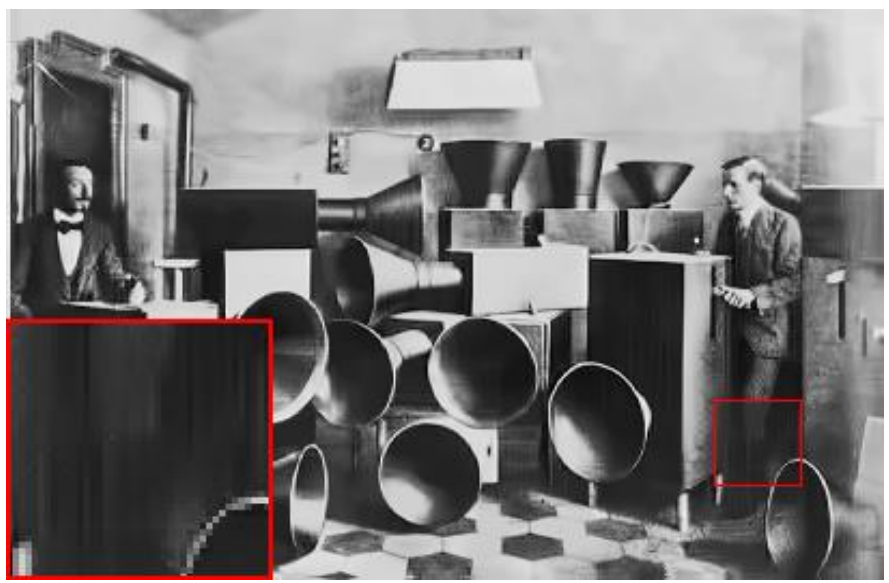

(b) BM3D

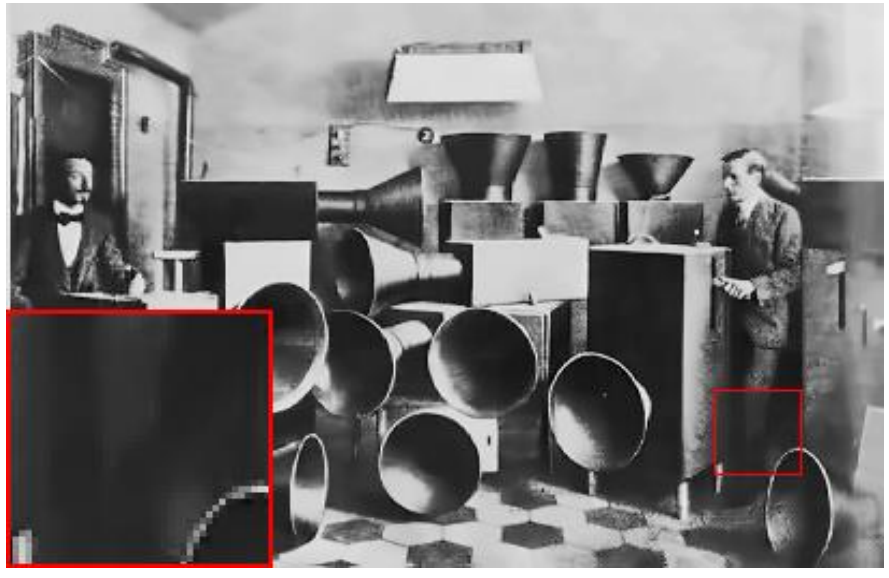

(d) GSRC-ENSS

Figure 10: Denoising results on real image by different methods. (a) Noisy image; (b) BM3D [12]; (c) PGPD [11; (d) GSRC-ENSS. 


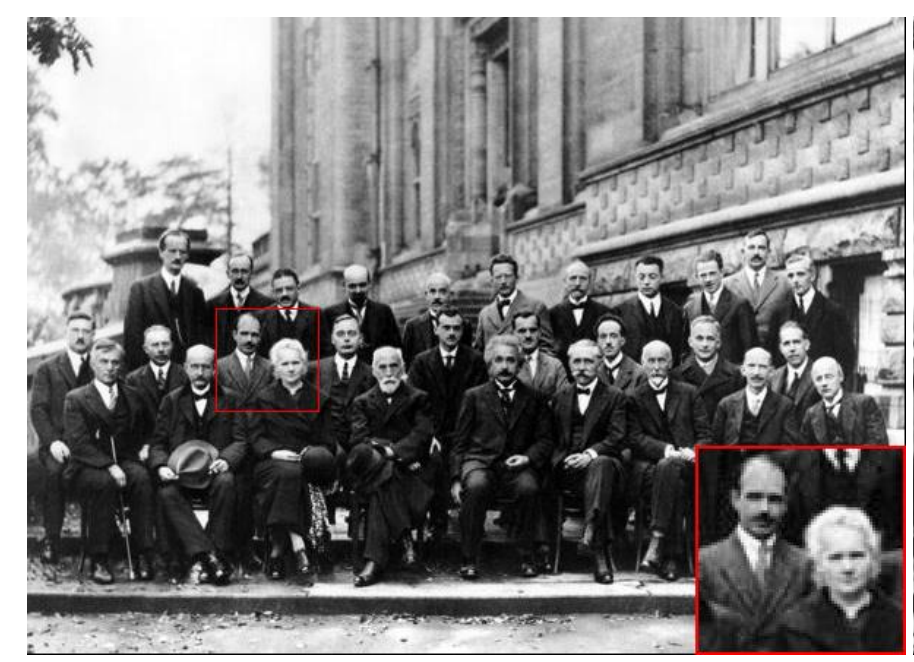

(a) Noisy image

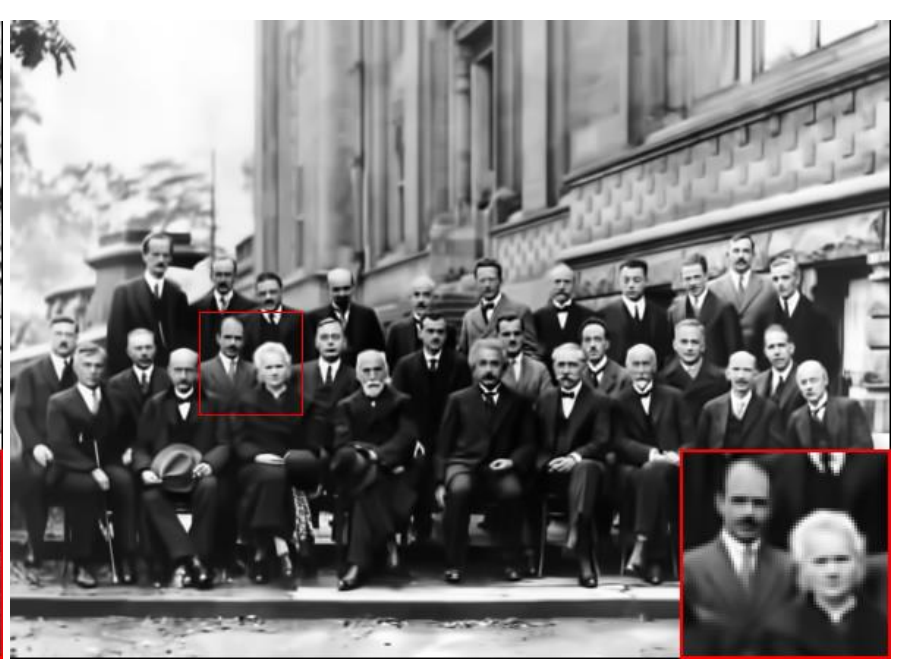

(b) BM3D

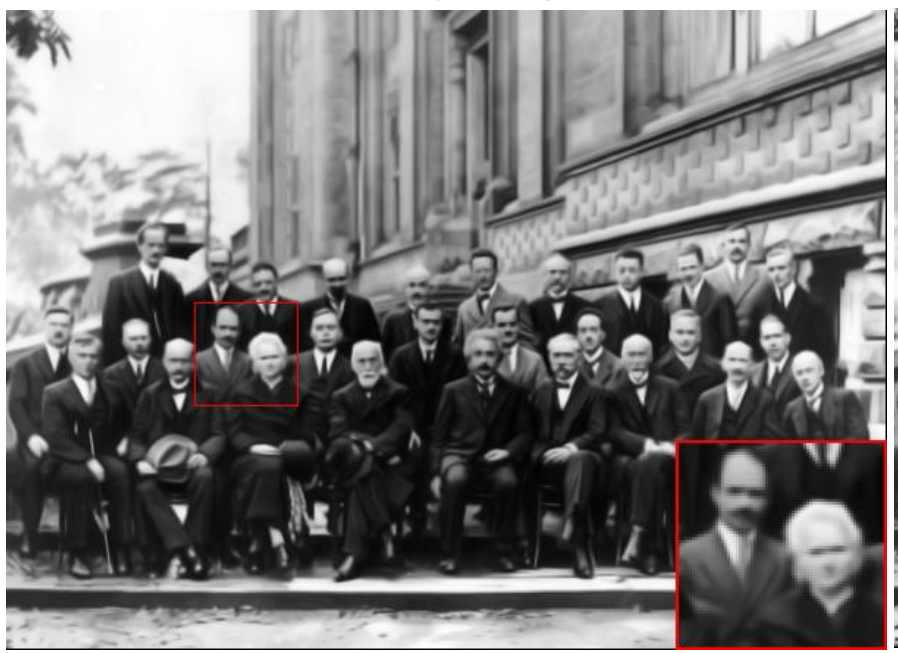

(c) PGPD

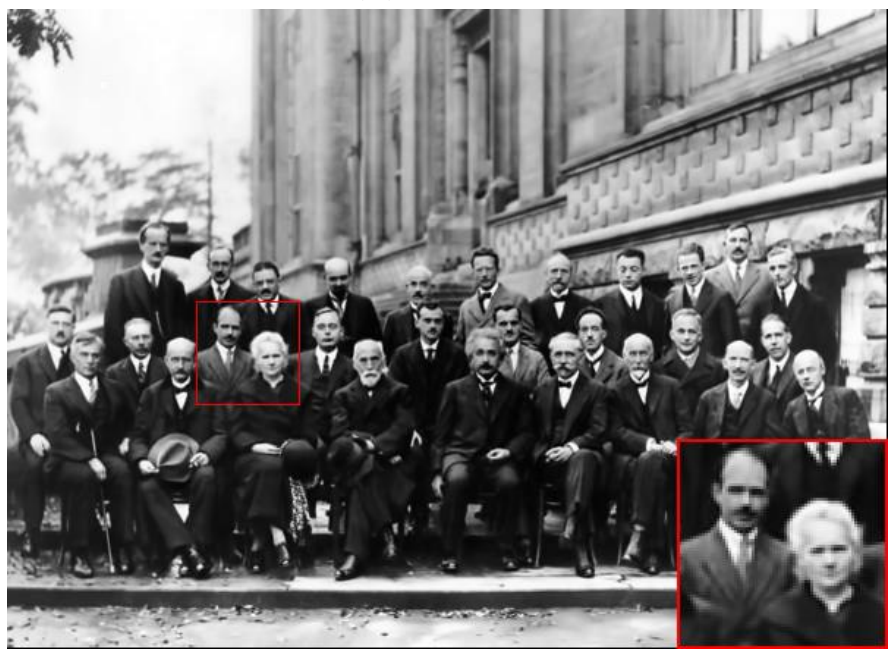

(d) GSRC-ENSS

Figure 11: Denoising results on real image by different methods. (a) Noisy image; (b) BM3D [12; (c) PGPD [11; (d) GSRC-ENSS. 
[4] Q. Dong, L. Li, Smooth incomplete matrix factorization and its applications in image/video denoising, Neurocomputing 122 (2013) 458-469.

[5] Y. Zhang, X. Tian, P. Ren, An adaptive bilateral filter based framework for image denoising, Neurocomputing 140 (2014) 299-316.

[6] M. Elad, M. Aharon, Image denoising via sparse and redundant representations over learned dictionaries, IEEE Transactions on Image processing 15 (12) (2006) 3736-3745.

[7] D. Zoran, Y. Weiss, From learning models of natural image patches to whole image restoration, in: Computer Vision (ICCV), 2011 IEEE International Conference on, IEEE, 2011, pp. 479-486.

[8] G. Yu, G. Sapiro, S. Mallat, Solving inverse problems with piecewise linear estimators: From gaussian mixture models to structured sparsity, IEEE Transactions on Image Processing 21 (5) (2012) 2481-2499.

[9] M. Niknejad, H. Rabbani, M. Babaie-Zadeh, Image restoration using gaussian mixture models with spatially constrained patch clustering, IEEE Transactions on Image Processing 24 (11) (2015) 3624-3636.

[10] B. Wen, S. Ravishankar, Y. Bresler, Structured overcomplete sparsifying transform learning with convergence guarantees and applications, Inप ternational Journal of Computer Vision 114 (2) (2015) 137-167. doi: 10.1007/s11263-014-0761-1. URL https://doi.org/10.1007/s11263-014-0761-1

[11] J. Xu, L. Zhang, W. Zuo, D. Zhang, X. Feng, Patch group based nonlocal self-similarity prior learning for image denoising, in: Proceedings of the IEEE International Conference on Computer Vision, 2015, pp. 244-252.

[12] K. Dabov, A. Foi, V. Katkovnik, K. Egiazarian, Image denoising by sparse 3-d transform-domain collaborative filtering, IEEE Transactions on image processing 16 (8) (2007) 2080-2095. 
[13] J. Mairal, F. Bach, J. Ponce, G. Sapiro, A. Zisserman, Non-local sparse models for image restoration, in: Computer Vision, 2009 IEEE 12th International Conference on, IEEE, 2009, pp. 2272-2279.

[14] L. Zhang, W. Dong, D. Zhang, G. Shi, Two-stage image denoising by principal component analysis with local pixel grouping, Pattern Recognition 43 (4) (2010) 1531-1549.

[15] T. Dai, C.-B. Song, J.-P. Zhang, S.-T. Xia, Pmpa: A patch-based multiscale products algorithm for image denoising, in: Image Processing (ICIP), 2015 IEEE International Conference on, IEEE, 2015, pp. 4406-4410.

[16] Q. Wang, X. Zhang, Y. Wu, L. Tang, Z. Zha, Nonconvex weighted $\ell_{p}$ minimization based group sparse representation framework for image denoising, IEEE Signal Processing Letters 24 (11) (2017) 1686-1690. doi: 10.1109/LSP. 2017.2731791

[17] B. Wen, S. Ravishankar, Y. Bresler, Frist-flipping and rotation invariant sparsifying transform learning and applications, Inverse Problems 33 (7) (2017) 074007.

URL http://stacks.iop.org/0266-5611/33/i=7/a=074007

[18] T. Dai, W. Lu, W. Wang, J. Wang, S.-T. Xia, Entropy-based bilateral filtering with a new range kernel, Signal Processing 137 (Supplement C) (2017) 223 - 234. doi:https://doi.org/10.1016/j.sigpro.2017.02.005 URL http://www.sciencedirect.com/science/article/pii/ S0165168417300518

[19] H. W. Engl, K. Kunisch, A. Neubauer, Convergence rates for tikhonov regularisation of non-linear ill-posed problems, Inverse problems 5 (4) (1989) 523.

[20] M. Aharon, M. Elad, A. Bruckstein, $k$-svd: An algorithm for designing overcomplete dictionaries for sparse representation, IEEE Transactions on signal processing 54 (11) (2006) 4311-4322. 
[21] J. Mairal, F. Bach, J. Ponce, G. Sapiro, Online dictionary learning for sparse coding, in: Proceedings of the 26th annual international conference on machine learning, ACM, 2009, pp. 689-696.

[22] J. Mairal, J. Ponce, G. Sapiro, A. Zisserman, F. R. Bach, Supervised dictionary learning, in: Advances in neural information processing systems, 2009, pp. 1033-1040.

[23] J. Zhang, D. Zhao, W. Gao, Group-based sparse representation for image restoration, IEEE Transactions on Image Processing 23 (8) (2014) 33363351.

[24] W. Dong, G. Shi, X. Li, Nonlocal image restoration with bilateral variance estimation: A low-rank approach, IEEE transactions on image processing 22 (2) (2013) 700-711.

[25] W. Dong, L. Zhang, G. Shi, X. Li, Nonlocally centralized sparse representation for image restoration, IEEE Transactions on Image Processing 22 (4) (2013) 1620-1630.

[26] R. Fergus, B. Singh, A. Hertzmann, S. T. Roweis, W. T. Freeman, Removing camera shake from a single photograph, in: ACM Transactions on Graphics (TOG), Vol. 25, ACM, 2006, pp. 787-794.

[27] W. Zuo, L. Zhang, C. Song, D. Zhang, H. Gao, Gradient histogram estimation and preservation for texture enhanced image denoising, IEEE Transactions on Image Processing 23 (6) (2014) 2459-2472.

[28] M. N. Do, M. Vetterli, Wavelet-based texture retrieval using generalized gaussian density and kullback-leibler distance, IEEE transactions on image processing 11 (2) (2002) 146-158.

[29] A. Rajwade, A. Rangarajan, A. Banerjee, Image denoising using the higher order singular value decomposition, IEEE Transactions on Pattern Analysis and Machine Intelligence 35 (4) (2013) 849-862. 
[30] W. Dong, L. Zhang, G. Shi, X. Wu, Image deblurring and super-resolution by adaptive sparse domain selection and adaptive regularization, IEEE Transactions on Image Processing 20 (7) (2011) 1838-1857.

[31] Q. Zhang, B. Li, Discriminative k-svd for dictionary learning in face recognition, in: Computer Vision and Pattern Recognition (CVPR), 2010 IEEE Conference on, IEEE, 2010, pp. 2691-2698.

[32] H. Zheng, D. Tao, Discriminative dictionary learning via fisher discrimination k-svd algorithm, Neurocomputing 162 (2015) 9-15.

[33] Z. Jiang, Z. Lin, L. S. Davis, Learning a discriminative dictionary for sparse coding via label consistent k-svd, in: Computer Vision and Pattern Recognition (CVPR), 2011 IEEE Conference on, IEEE, 2011, pp. 1697-1704.

[34] D. P. Lun, et al., Robust fringe projection profilometry via sparse representation, IEEE Transactions on Image Processing 25 (4) (2016) 1726-1739.

[35] Z. Zha, X. Liu, X. Zhang, Y. Chen, L. Tang, Y. Bai, Q. Wang, Z. Shang, Compressed sensing image reconstruction via adaptive sparse nonlocal regularization, The Visual Computer (2016) 1-21.

[36] H. Ji, C. Liu, Z. Shen, Y. Xu, Robust video denoising using low rank matrix completion, in: Computer Vision and Pattern Recognition (CVPR), 2010 IEEE Conference on, IEEE, 2010, pp. 1791-1798.

[37] S. Gu, L. Zhang, W. Zuo, X. Feng, Weighted nuclear norm minimization with application to image denoising, in: Proceedings of the IEEE Conference on Computer Vision and Pattern Recognition, 2014, pp. 2862-2869.

[38] J. Zhang, D. Zhao, R. Xiong, S. Ma, W. Gao, Image restoration using joint statistical modeling in a space-transform domain, IEEE Transactions on Circuits and Systems for Video Technology 24 (6) (2014) 915-928.

[39] I. Mosseri, M. Zontak, M. Irani, Combining the power of internal and external denoising, in: Computational Photography (ICCP), 2013 IEEE International Conference on, IEEE, 2013, pp. 1-9. 
[40] H. Yue, X. Sun, J. Yang, F. Wu, Image denoising by exploring external and internal correlations, IEEE Transactions on Image Processing 24 (6) (2015) 1967-1982.

[41] X. Lu, Z. Lin, H. Jin, J. Yang, J. Z. Wang, Image-specific prior adaptation for denoising, IEEE Transactions on Image Processing 24 (12) (2015) 54695478 .

[42] E. Luo, S. H. Chan, T. Q. Nguyen, Adaptive image denoising by mixture adaptation, IEEE Transactions on Image Processing 25 (10) (2016) 44894503.

[43] V. Jain, S. Seung, Natural image denoising with convolutional networks, in: Advances in Neural Information Processing Systems, 2009, pp. 769-776.

[44] Y. Chen, T. Pock, Trainable nonlinear reaction diffusion: A flexible framework for fast and effective image restoration, IEEE transactions on pattern analysis and machine intelligence 39 (6) (2017) 1256-1272.

[45] H. C. Burger, C. J. Schuler, S. Harmeling, Image denoising: Can plain neural networks compete with bm3d?, in: Computer Vision and Pattern Recognition (CVPR), 2012 IEEE Conference on, IEEE, 2012, pp. 23922399 .

[46] S. Liu, J. Pan, M.-H. Yang, Learning recursive filters for low-level vision via a hybrid neural network, in: European Conference on Computer Vision, Springer, 2016, pp. 560-576.

[47] K. Zhang, W. Zuo, Y. Chen, D. Meng, L. Zhang, Beyond a gaussian denoiser: Residual learning of deep cnn for image denoising, IEEE Transactions on Image Processing.

[48] X. Mao, C. Shen, Y.-B. Yang, Image restoration using very deep convolutional encoder-decoder networks with symmetric skip connections, in: Advances in Neural Information Processing Systems, 2016, pp. 2802-2810. 
[49] G. R. Cross, A. K. Jain, Markov random field texture models, IEEE Transactions on Pattern Analysis and Machine Intelligence (1) (1983) 25-39.

[50] W. Dong, G. Shi, Y. Ma, X. Li, Image restoration via simultaneous sparse coding: Where structured sparsity meets gaussian scale mixture, International Journal of Computer Vision 114 (2-3) (2015) 217-232.

[51] Z. Zha, X. Liu, X. Huang, H. Shi, Y. Xu, Q. Wang, L. Tang, X. Zhang, Analyzing the group sparsity based on the rank minimization methods, in: 2017 IEEE International Conference on Multimedia and Expo (ICME), 2017, pp. 883-888. doi:10.1109/ICME.2017.8019334.

[52] X. Li, H. He, R. Wang, D. Tao, Single image superresolution via directional group sparsity and directional features, IEEE Transactions on Image Processing 24 (9) (2015) 2874-2888.

[53] Z. Zha, X. Liu, Z. Zhou, X. Huang, J. Shi, Z. Shang, L. Tang, Y. Bai, Q. Wang, X. Zhang, Image denoising via group sparsity residual constraint, International Conference on Acoustics, Speech and Signal Processing (ICASSP), 2017.

[54] C. M. Bishop, Pattern recognition, Machine Learning 128 (2006) 1-58.

[55] P. Sandeep, T. Jacob, Single image super-resolution using a joint gmm method, IEEE Transactions on Image Processing 25 (9) (2016) 4233-4244.

[56] I. Daubechies, M. Defrise, C. De Mol, An iterative thresholding algorithm for linear inverse problems with a sparsity constraint, Communications on pure and applied mathematics 57 (11) (2004) 1413-1457.

[57] S. G. Chang, B. Yu, M. Vetterli, Adaptive wavelet thresholding for image denoising and compression, IEEE Transactions on image processing 9 (9) (2000) 1532-1546.

[58] S. Osher, M. Burger, D. Goldfarb, J. Xu, W. Yin, An iterative regularization method for total variation-based image restoration, Multiscale Modeling \& Simulation 4 (2) (2005) 460-489. 
[59] G. Peyré, Image processing with nonlocal spectral bases, Multiscale Modeling \& Simulation 7 (2) (2008) 703-730.

[60] X. Zhang, M. Burger, X. Bresson, S. Osher, Bregmanized nonlocal regularization for deconvolution and sparse reconstruction, SIAM Journal on Imaging Sciences 3 (3) (2010) 253-276.

[61] H. C. Burger, C. Schuler, S. Harmeling, Learning how to combine internal and external denoising methods, in: German Conference on Pattern Recognition, Springer, 2013, pp. 121-130.

[62] F. Chen, L. Zhang, H. Yu, External patch prior guided internal clustering for image denoising, in: Proceedings of the IEEE International Conference on Computer Vision, 2015, pp. 603-611.

[63] K. Tibell, H. Spies, M. Borga, Fast prototype based noise reduction, in: Scandinavian Conference on Image Analysis, Springer, 2009, pp. 159-168.

[64] H. Talebi, P. Milanfar, Global image denoising, IEEE Transactions on Image Processing 23 (2) (2014) 755-768.

[65] Z. Wang, A. C. Bovik, H. R. Sheikh, E. P. Simoncelli, Image quality assessment: from error visibility to structural similarity, IEEE transactions on image processing 13 (4) (2004) 600-612. 